\title{
Old open clusters in the outer Galactic disk ${ }^{\star} \star \star$
}

\author{
G. Carraro ${ }^{1,2}$, D. Geisler ${ }^{3}$, S. Villanova ${ }^{1}$, P. M. Frinchaboy ${ }^{4}$, and S. R. Majewski ${ }^{5}$ \\ 1 Dipartimento di Astronomia, Università di Padova, Vicolo Osservatorio 2, 35122 Padova, Italy \\ e-mail: giovanni . carraro, sandro.villanova@unipd.it \\ 2 ESO, Alonso de Cordova, 3107 Vitacura, Santiago de Chile, Chile \\ e-mail: gcarraro@eso.org \\ 3 Universidad de Concepción, Departamento de Fisica, Casilla 160-C, Concepción, Chile \\ e-mail: dgeisler@astro-udec.cl \\ 4 NSF Astronomy and Astrophysics Postdoctoral Fellow \\ University of Wisconsin-Madison, Department of Astronomy, 475 N. Charter Street, Madison, WI 53706, USA \\ e-mail: pmf@astro.wisc.edu \\ 5 Department of Astronomy, University of Virginia, PO Box 400325, Charlottesville, VA 22903-4325, USA \\ e-mail: srm4n@virginia.edu
}

Received 19 June 2007 / Accepted 10 September 2007

\begin{abstract}
Context. The outer parts of the Milky Way disk are believed to be one of the main arenas where the accretion of external material in the form of dwarf galaxies and subsequent formation of streams is taking place. The Monoceros stream and the Canis Major and Argo over-densities are notorious examples. Understanding whether what we detect is the signature of accretion or, more conservatively, simply the intrinsic nature of the disk, represents one of the major goals of modern Galactic astronomy.

Aims. We try to shed more light on the properties of the outer disk by exploring the properties of distant anti-center old open clusters. We want to verify whether distant clusters follow the chemical and dynamical behavior of the solar vicinity disk, or whether their properties can be better explained in terms of an extra-galactic population.

Methods. VLT high resolution spectra have been acquired for five distant open clusters: Ruprecht 4, Ruprecht 7, Berkeley 25, Berkeley 73 and Berkeley 75 . We derive accurate radial velocities to distinguish field interlopers and cluster members. For the latter we perform a detailed abundance analysis and derive the iron abundance $[\mathrm{Fe} / \mathrm{H}]$ and the abundance ratios of several $\alpha$ elements.

Results. Our analysis confirms previous indications that the radial abundance gradient in the outer Galactic disk does not follow the expectations extrapolated from the solar vicinity, but exhibits a shallower slope. By combining the metallicity of the five program clusters with eight more clusters for which high resolution spectroscopy is available, we find that the mean metallicity in the outer disk between 12 and $21 \mathrm{kpc}$ from the Galactic center is $[\mathrm{Fe} / \mathrm{H}] \approx-0.35$, with only marginal indications for a radial variation. In addition, all the program clusters exhibit solar scaled or slightly enhanced $\alpha$ elements, similar to open clusters in the solar vicinity and thin disk stars.

Conclusions. We investigate whether this outer disk cluster sample might belong to an extra-galactic population, like the Monoceros ring. However, close scrutiny of their properties - location, kinematics and chemistry - does not convincingly favor this hypothesis. On the contrary, they appear more likely genuine Galactic disk clusters. We finally stress the importance to obtain proper motion measurements for these clusters to constrain their orbits.
\end{abstract}

Key words. open clusters and associations: general - stars: fundamental parameters - Galaxy: disk -

Galaxy: evolution - Galaxy: structure

\section{Introduction}

While there are much data on chemical abundances in the solar vicinity both for disk stars (Ramírez et al. 2007; Bensby et al. 2004) and for star clusters (Friel et al. 2002), our knowledge of the outer Galactic disk is still very poor. This is particularly true for Galactic open clusters, which are known to extend to very far in the disk periphery, but whose data in many cases basically consist of shallow photometry which only allows us to derive rough estimates of age and distance (e.g., Phelps et al. 1994; Frinchaboy \& Phelps 2002; Carraro et al. 2006).

\footnotetext{
* Based on observations with the ESO VLT at the Paranal Observatory, under the program 076.B-0263.

$\star \star$ Table 3 is only available in electronic form at http://www. aanda.org
}

In the last few years the situation has improved thanks to a renewal of interest in the outer Galactic disk which, according to several studies, may have been formed though several mergers of small galaxies. In this context, a structure like the Monoceros ring (hereinafter MRi, Newberg et al. 2002; Ibata et al. 2003; Crane et al. 2003; Rocha-Pinto et al. 2003) would be the best signature of such accretions. Distant star clusters - both open and globular - may trace this structure (Frinchaboy et al. 2004, 2006), date its formation time, and probe its chemical evolution, but the lack of precise metallicity, distance and age data for a sufficient number of potentially associated clusters makes their connection to such over-densities still vague.

Recently, good abundance data have started to be acquired for a handful of distant clusters (Carraro et al. 2004; Yong et al. 2005; Villanova et al. 2005; Frinchaboy et al. 2007). All these 
studies seem to indicate that the outer disk does not follow closely the chemical pattern one would expect from an extrapolation of the solar neighborhood data. For instance, the radial $[\mathrm{Fe} / \mathrm{H}]$ gradient, instead of relatively steeply declining as in the solar vicinity, deviates at $R_{\mathrm{GC}}>10-12 \mathrm{kpc}$ and stays almost flat toward the Galactic anti-center, while the $\alpha$ elements are moderately enhanced with respect to the Sun. The metallicity flattening by the way was suggested as early as the study of Twarog et al. (1997), but with a largely inhomogeneous data-set.

These trends are seen in other tracers, like field giants and Cepheids (Carney et al. 2005; Yong et al. 2006). But while these chemical characteristics may cast new light on the formation of the outer Galactic disk, in fact the number of anti-center tracers with good abundance data, particularly distant star clusters, remains too small to attempt reliable speculations, despite their obvious statistical advantage for deriving ages, kinematics, abundances, and so forth.

Meanwhile, current models of the chemical evolution of the Galactic disk (e.g., Cescutti et al. 2006, and references therein) have started to predict the radial abundance gradients for several elements in the outer regions of the Milky Way. While these models employ updated prescriptions for all of the basic ingredients (reactions rates, Initial Mass Function and so forth) of the calculation - and in this respect are very sophisticated and have high predictive power - again the lack of sufficient data for elemental abundances in the outer disk prevents careful comparisons with observations and limits the applicability of the models.

In an attempt to improve this situation, we present in this paper the results of a spectroscopic campaign conducted with the Very Large Telescope (VLT) of five previously unstudied, old and distant open clusters toward the Galactic anti-center. These particular clusters were targeted to help clarify their status as genuine disk clusters or as possible members of the MRi. At the same time, our data are intended to provide a better set of observational templates for chemical evolution models.

\section{Selection of clusters}

The clusters under investigation are listed in Table 1, together with their Galactic coordinates and preliminary estimates of distance and age as reported in Carraro et al. (2005a,b). In those papers, BVI photometry has been obtained with the purpose of searching for potentially old and distant clusters and providing targets for later spectroscopic follow-up.

Together with these five clusters we have also observed Tombaugh 2, which we report on elsewhere (Frinchaboy et al. 2007). We elected to study these targets in detail because we wanted to probe a region of the Milky Way - the Third Galactic Quadrant - where the signatures of ongoing accretions (the putative Canis Major galaxy, hereafter "CMa", and the MRi) have been repeatedly pointed out, but where other explanations are also possible. Open clusters can help us to shed more light on the structure and origin of the outer disk because of the ability to derive accurate determinations of age, distance, radial velocity, metallicity, and detailed abundances for these systems. Establishing any relationships between position and kinematics and/or age and metallicity is the first step not only to better describe the chemical and dynamical evolution of the Galactic disk periphery, but also to recognize possible structures not associated with the Galactic disk (Frinchaboy et al. 2004).

For our study we wanted a sample of clusters covering a wide baseline in Galactocentric distance, older than the Hyades,
Table 1. Clusters sample.

\begin{tabular}{cccccc}
\hline \hline Name & $l$ & $b$ & $d_{\odot}$ & age & Ref. \\
\hline & deg & deg & $\mathrm{kpc}$ & Gyr & \\
\hline Berkeley 75 & 234.30 & -11.12 & 9.8 & 3.0 & Carraro et al. (2005a) \\
Berkeley 25 & 226.60 & -9.69 & 11.3 & 4.0 & Carraro et al. (2005a) \\
Ruprecht 7 & 225.44 & -4.58 & 6.5 & 0.8 & Carraro et al. (2005b) \\
Ruprecht 4 & 222.04 & -5.31 & 4.9 & 0.8 & Carraro et al. (2005b) \\
Berkeley 73 & 215.28 & -9.42 & 9.7 & 1.5 & Carraro et al. (2005a) \\
\hline
\end{tabular}

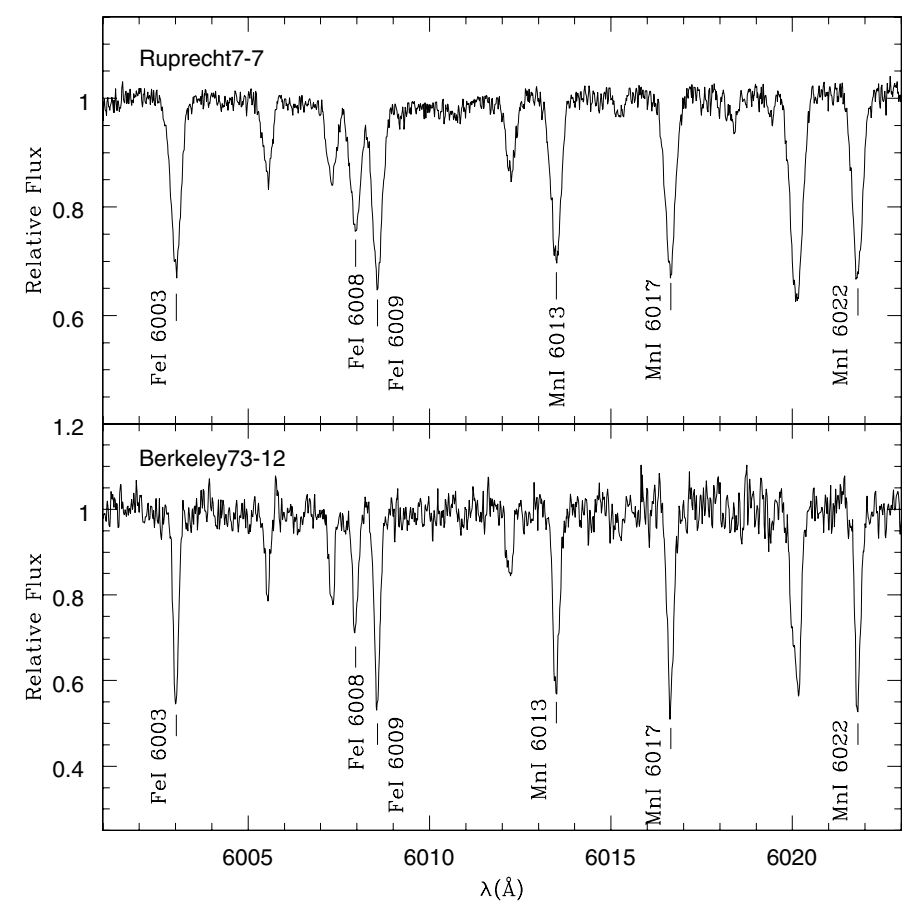

Fig. 1. The spectrum of Ruprecht 7-7 (upper panel) and Berkeley 73-12 (lower panel) in the wave-length interval 6000-6025 A. Several spectral lines are identified.

located toward the CMa and MRi over-densities and below the plane to further investigate the disk warp (Momany et al. 2006).

All five of the Table 1 clusters are projected toward the Monoceros/Canis Major constellations in the southern Galactic hemisphere. Indeed, they are all projected onto or very near the Canis Majoris overdensity itself (see Moitinho et al. 2006). They also all lie significantly below the nominal Galactic plane. According to the preliminary analysis by Carraro et al. (2005a,b), they presently all lie beyond $12 \mathrm{kpc}$ from the Galactic center and are likely older than the Hyades (see Table 1).

To our knowledge, no previous estimates of radial velocity or metallicity are available for any of these clusters.

\section{Observations and data reduction}

The spectroscopic data come from the ESO-VLT, and were collected in service mode between October and December 2005 with the FLAMES on-board VLT+UVES high-resolution, echelle spectrograph. The sky was generally clear, and the typical seeing was about 0.8 arcsec during all of the observing nights. We used the UVES multifiber mode, which allows the collection of up to eight spectra simultaneously. The $580 \mathrm{~nm}$ setup ( $R=40000$ in the 4750-6800 $\AA$ range) was used. The UVES fibers were placed on the brighter probable members of the cluster according to their CMD position. The data were reduced by 
Table 2. Radial velocities plus photometry of the program stars.ID is identification according to Carraro et al. (2005a,b) numbering. The last column indicates whether a star is considered member (M) or not (NM) according to the analysis in Sect. 5.

\begin{tabular}{|c|c|c|c|c|c|c|c|c|c|}
\hline Cluster & $\overline{\mathrm{ID}}$ & $\alpha(2000.0)$ & $\overline{\delta(2000.0)}$ & $\overline{\bar{B}}$ & $\overline{\bar{V}}$ & $\bar{I}$ & $\bar{R} R V_{\mathrm{H}}$ & 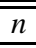 & Membership \\
\hline & & hh:mm:s & $o::^{\prime \prime}$ & & \multicolumn{5}{|c|}{$\left[\mathrm{km} \mathrm{s}^{-1}\right]$} \\
\hline \multirow[t]{2}{*}{ Berkeley 75} & 9 & 06:49:07.09 & $-23: 59: 44.94$ & 15.78 & 14.89 & 13.71 & $73.10 \pm 0.50$ & 2 & NM \\
\hline & 22 & $06: 48: 55.85$ & $-24: 00: 07.16$ & 17.08 & 16.13 & 14.99 & $94.60 \pm 0.35$ & 2 & M \\
\hline \multirow[t]{5}{*}{ Berkeley 25} & 10 & $06: 41: 20.40$ & $-16: 28: 23.63$ & 17.45 & 16.13 & 14.56 & $134.10 \pm 0.20$ & 2 & NM \\
\hline & 12 & $06: 41: 16.60$ & $-16: 28: 16.13$ & 17.59 & 16.32 & 14.78 & $135.60 \pm 0.20$ & 2 & M \\
\hline & 13 & $06: 41: 13.63$ & $-16: 29: 17.27$ & 17.36 & 16.33 & 14.97 & $133.30 \pm 0.20$ & 2 & M \\
\hline & 21 & 06:41:08.37 & $-16: 29: 20.13$ & 17.59 & 16.50 & 15.08 & $134.00 \pm 0.50$ & 2 & M \\
\hline & 23 & $06: 41: 16.76$ & $-16: 29: 21.34$ & 17.91 & 16.72 & 15.27 & $131.70 \pm 0.50$ & 2 & M \\
\hline \multirow[t]{5}{*}{ Ruprecht 7} & 2 & $06: 57: 50.14$ & $-13: 13: 39.93$ & 15.47 & 14.07 & 12.71 & $77.12 \pm 0.20$ & 3 & M \\
\hline & 4 & $06: 57: 43.08$ & $-13: 12: 17.41$ & 16.02 & 14.58 & 12.71 & $77.10 \pm 0.25$ & 3 & M \\
\hline & 5 & 06:57:54.56 & $-13: 13: 59.18$ & 16.17 & 14.78 & 12.95 & $77.12 \pm 0.20$ & 3 & M \\
\hline & 6 & 06:57:53.66 & $-13: 12: 57.83$ & 16.24 & 14.79 & 12.91 & $77.61 \pm 0.30$ & 3 & M \\
\hline & 7 & $06: 57: 52.85$ & $-13: 12: 55.08$ & 16.41 & 15.07 & 12.25 & $74.26 \pm 0.90$ & 3 & M \\
\hline \multirow[t]{5}{*}{ Ruprecht 4} & 3 & 06:48:51.69 & $-10: 30: 20.49$ & 15.46 & 14.24 & 12.69 & $48.40 \pm 1.00$ & 1 & M \\
\hline & 4 & 06:49:00.80 & $-10: 31: 57.81$ & 15.60 & 14.30 & 12.68 & $45.85 \pm 0.55$ & 2 & M \\
\hline & 8 & $06: 48: 55.37$ & $-10: 31: 32.39$ & 15.94 & 14.77 & 13.26 & $48.40 \pm 1.00$ & 1 & M \\
\hline & 18 & $06: 48: 54.51$ & $-10: 33: 21.32$ & 16.62 & 15.44 & 13.90 & $62.20 \pm 1.00$ & 1 & NM \\
\hline & 29 & 06:48:57.87 & $-10: 30: 27.09$ & 17.29 & 15.95 & 14.25 & $62.20 \pm 1.00$ & 1 & NM \\
\hline \multirow[t]{6}{*}{ Berkeley 73} & 12 & $06: 21: 54.89$ & $-06: 17: 51.91$ & 16.30 & $15: 30$ & 13.93 & $89.10 \pm 0.20$ & 2 & NM \\
\hline & 13 & $06: 21: 55.36$ & $-06: 20: 10.04$ & 16.10 & 15.36 & 14.30 & $75.35 \pm 0.25$ & 2 & NM \\
\hline & 15 & $06: 22: 10.44$ & $-06: 19: 30.19$ & 16.51 & 15.57 & 14.30 & $73.30 \pm 0.30$ & 2 & NM \\
\hline & 16 & 06:22:01.13 & $-06: 19: 17.14$ & 16.86 & 15.84 & 14.48 & $95.30 \pm 0.20$ & 2 & M \\
\hline & 18 & 06:22:01.99 & $-06: 19: 21.89$ & 17.10 & 15.99 & 14.58 & $96.10 \pm 0.20$ & 2 & M \\
\hline & 19 & $06: 21: 57.24$ & $-06: 17: 15.55$ & 16.74 & 16.03 & 14.95 & $92.85 \pm 0.25$ & 2 & NM \\
\hline
\end{tabular}

ESO personnel using the FLAMES-UVES pipeline ${ }^{1}$ which first corrects the spectra for bias and flatfield. Next a wavelength calibration based on Th-Ar calibration-lamp spectra was applied. Finally, each spectrum is flux-calibrated applying the responsecurve of the instrument and the echelle orders combined to obtain a single one-dimensional spectrum. The resulting spectra have a dispersion of $0.1 \AA /$ pixel and a typical $S / N \sim 25-40$. We could observe 21 Red Giant Branch(RGB)/red clump stars in the five program clusters, for a grand total of 43 spectra. Due to the small size of the clusters under study, it was not possible to put all eight fibers on cluster stars. Typically we could configure UVES to pick up five stars per cluster. In the case of Berkeley 75 we could observe just two stars with sufficiently high $S / N$. Table 2 provides information on the targets including photometry from Carraro et al. (2005a,b). Note that we obtained $1-3$ independent spectra ( $n$, Col. 9 in Table 2) per star, and the integration time was $45 \mathrm{~min}$ per exposure. Examples of extracted spectra are illustrated in Fig. 1, where the spectrum of star \#7 in Ruprecht 7 and \#12 in Berkeley 73 are shown.

\subsection{Radial velocities}

We used the fxcor IRAF utility to measure radial velocities. This routine cross-correlates the observed spectrum with a template having known radial velocity. As a template we used a synthetic spectrum calculated for a typical solar metallicity giant $\operatorname{star}\left(T_{\text {eff }} \sim 5000 \mathrm{~K}, \log (g)=3.0, v_{\mathrm{t}}=1.3 \mathrm{~km} \mathrm{~s}^{-1}\right)$. Then each measured radial velocity was converted to heliocentric velocity using the rvcorr IRAF routine. The resulting heliocentric velocities for the stars $\left(R V_{\mathrm{H}}\right)$ are reported in Table 2 . The error in radial velocity - derived from repeated measures - is less than

${ }^{1}$ See http://www.eso.org/projects/dfs/dfs-shared/ web/vlt/vlt-instrument-pipelines.html for documentation on the FLAMES-UVES pipeline and software.
$1 \mathrm{~km} \mathrm{~s}^{-1}$. Finally, for the abundance analysis each spectrum was shifted to rest-frame velocity and continuum-normalized.

\section{Abundance analysis}

\subsection{Atomic parameters and equivalent widths}

The analysis of chemical abundances was carried out with the 2005 version of the freely available program MOOG developed by Chris Sneden ${ }^{2}$ and originally described in Sneden (1973) and using model atmospheres by Kurucz (1992). MOOG performs a local thermodynamical equilibrium (LTE) analysis. We derived equivalent widths by fitting Gaussian profiles to spectral lines. Repeated measurements show a typical error of about 5-10 $\mathrm{m} \AA$ for the weakest lines because of the moderate $S / N$ of the spectra. The line list (see Table 3) was taken from Gratton et al. (2003). The $\log (g f)$ parameters of these lines were redetermined by a solar-inverse analysis measuring the equivalent widths from the NOAO (Kurucz et al. 1984) solar spectrum, adopting the standard solar parameters $\left(T_{\mathrm{eff}}=5777 \mathrm{~K}\right.$, $\left.\log (g)=4.44, v_{\mathrm{t}}=0.8 \mathrm{~km} \mathrm{~s}^{-1}, A(\mathrm{Fe})=7.48\right)$.

\subsection{Atmospheric parameters}

Initial estimates of the atmospheric parameter $T_{\text {eff }}$ were obtained from the photometric observations given in Table 2 using the relations from Alonso et al. (1999). We adopted $E(B-V)$ values from Carraro et al. $(2005 a, b)$ to correct colours for the interstellar extinction. We then adjusted the effective temperature by minimizing the slope of the abundances obtained from Fe I lines with respect to the excitation potential. Initial guesses for the gravity $\log (g)$ were derived from the canonical formula:

$\log \left(\frac{g}{g_{\odot}}\right)=\log \left(\frac{M}{M_{\odot}}\right)+4 \log \left(\frac{T_{\text {eff }}}{T_{\odot}}\right)-\log \left(\frac{L}{L_{\odot}}\right)$.

\footnotetext{
${ }^{2}$ http://verdi.as.utexas.edu/moog.html
} 
Table 4. Atmospheric parameters. Typical errors in temperature, logarithm of gravity and micro-turbulent velocity are $100^{\circ} \mathrm{K}, 0.2$, and $0.1 \mathrm{~km} \mathrm{~s}^{-1}$.

\begin{tabular}{|c|c|c|c|c|c|c|c|}
\hline Cluster & ID & $T_{\text {eff }}$ & $\log (g)$ & $v_{\mathrm{t}}$ & $\log (\mathrm{Fe})$ & {$[\mathrm{Fe} / \mathrm{H}]$} & Membership \\
\hline & & ${ }^{\circ} \mathrm{K}$ & & $\mathrm{km} \mathrm{s}^{-1}$ & & dex & \\
\hline \multirow[t]{2}{*}{ Berkeley 75} & 9 & 4968 & 2.57 & 1.55 & 7.04 & $-0.44 \pm 0.03$ & NM \\
\hline & 22 & 5180 & 3.37 & 1.21 & 7.26 & $-0.22 \pm 0.03$ & M \\
\hline \multirow[t]{5}{*}{ Berkeley 25} & 10 & 5000 & 2.90 & 1.65 & 7.58 & $+0.10 \pm 0.05$ & NM \\
\hline & 12 & 4870 & 2.75 & 1.50 & 7.28 & $-0.20 \pm 0.04$ & M \\
\hline & 13 & 4860 & 2.65 & 1.73 & 7.31 & $-0.17 \pm 0.04$ & M \\
\hline & 21 & 5100 & 2.50 & 1.80 & 7.32 & $-0.19 \pm 0.14$ & M \\
\hline & 23 & 5000 & 2.50 & 1.80 & 7.26 & $-0.25 \pm 0.12$ & M \\
\hline \multirow[t]{5}{*}{ Ruprecht 7} & 2 & 5160 & 2.12 & 1.62 & 7.14 & $-0.34 \pm 0.02$ & M \\
\hline & 4 & 5105 & 2.05 & 1.90 & 7.24 & $-0.24 \pm 0.01$ & M \\
\hline & 5 & 5230 & 2.19 & 2.10 & 7.21 & $-0.27 \pm 0.01$ & M \\
\hline & 6 & 5230 & 2.23 & 2.08 & 7.28 & $-0.20 \pm 0.01$ & M \\
\hline & 7 & 5150 & 2.40 & 1.82 & 7.23 & $-0.25 \pm 0.01$ & M \\
\hline \multirow[t]{5}{*}{ Ruprecht 4} & 3 & 5180 & 2.63 & 1.56 & 7.41 & $-0.07 \pm 0.01$ & M \\
\hline & 4 & 5150 & 2.52 & 1.66 & 7.45 & $-0.04 \pm 0.02$ & M \\
\hline & 8 & 5190 & 2.64 & 1.40 & 7.32 & $-0.16 \pm 0.01$ & M \\
\hline & 18 & 5040 & 3.17 & 1.20 & 7.13 & $-0.35 \pm 0.01$ & NM \\
\hline & 29 & 4920 & 2.78 & 1.37 & 7.14 & $-0.34 \pm 0.02$ & NM \\
\hline \multirow[t]{6}{*}{ Berkeley 73} & 12 & 5030 & 2.78 & 1.40 & 7.09 & $-0.39 \pm 0.02$ & NM \\
\hline & 13 & 5730 & 4.15 & 0.99 & 7.65 & $+0.17 \pm 0.01$ & NM \\
\hline & 15 & 5070 & 3.12 & 1.04 & 7.10 & $-0.38 \pm 0.01$ & NM \\
\hline & 16 & 4890 & 2.71 & 1.45 & 7.30 & $-0.18 \pm 0.02$ & M \\
\hline & 18 & 4940 & 2.88 & 1.32 & 7.23 & $-0.27 \pm 0.02$ & M \\
\hline & 19 & 5870 & 4.23 & 1.40 & 7.45 & $-0.03 \pm 0.03$ & NM \\
\hline
\end{tabular}

In this equation, the mass $M / M_{\odot}$ was derived from the comparison between the position of the star in the Hertzsprung-Russell diagram and the Padova isochrones (Girardi et al. 2000). The luminosity $L / L_{\odot}$ was derived from the apparent magnitude $V$, assuming the distance moduli from Carraro et al. $(2005 \mathrm{a}, \mathrm{b})$ The bolometric correction (BC) was derived from the $\mathrm{BC}-T_{\text {eff }}$ relation from Alonso et al. (1999). The input $\log (g)$ values were then adjusted to satisfy the ionization equilibrium of $\mathrm{Fe} \mathrm{I}$ and $\mathrm{Fe}$ II during the abundance analysis. Finally, the micro-turbulence velocity is given by the relation (Houdashelt et al. 2000): $v_{\mathrm{t}}=$ $2.22-0.322 \log g$. We then adjusted the micro-turbulence velocity by minimizing the slope of the abundances obtained from Fe I lines. The adopted values for all these parameters together with derived $[\mathrm{Fe} / \mathrm{H}]$ are reported in Table 4 , whereas the mean results of the detailed abundance analysis per cluster (i.e. with results averaged over all adopted cluster members - see Sect. 5) are listed in Table 5. We also report there our measures of the main $\alpha$ elements with their uncertainties and abundance ratios.

We made a detailed error analysis and found that an increase of 0.1 in micro-turbulence velocity implies an increase of $0.01 \mathrm{dex}$ in any of the measured elements ( $\mathrm{Fe}, \mathrm{Mg}, \mathrm{Si}, \mathrm{Ca}$ and $\mathrm{Ti})$. On the other hand, a variation of 0.2 in $\log (g)$ produces a decrease of 0.01 dex in any of the elements. Much more sensitive is the dependence on temperature. An increase of $100^{\circ} \mathrm{K}$ produces variations as large as $+0.11,+0.08,+0.06,+0.10$, and +0.16 dex in $\mathrm{Fe}, \mathrm{Mg}, \mathrm{Si}, \mathrm{Ca}$, and $\mathrm{Ti}$, respectively.

\section{Revision of cluster fundamental parameters}

We make use here of the derived radial velocity, metallicity, and available photometry to revise the fundamental parameters of the clusters under investigation.

First, we discuss the membership of all the program stars on the basis of their radial velocity (Table 2), position in the CMD, location in the cluster field, atmospheric parameters, and chemistry. From the stars that have been selected as members, we compute the cluster mean metal abundance (see Table 5). We use the following criterion to add error bars to the cluster mean iron abundance $[\mathrm{Fe} / \mathrm{H}]$. We adopt as metallicity error 0.2 dex when cluster metallicity is based on just 1 member, 0.1 dex in the case we find two members, and 0.05 dex when we have 3 or more members, but only in the case the measurements standard deviation $\sigma$ is smaller than this value, otherwise we directly adopt the measurements $\sigma$.

We then generate isochrones for the derived spectroscopic metallicity of each cluster, transforming the mean $[\mathrm{Fe} / \mathrm{H}]$ into $Z$, using Padova models and following Carraro et al. (1999). Owing to the marginal $\alpha$-element enhancement, we derived $Z$ considering only $[\mathrm{Fe} / \mathrm{H}]$. The corresponding best-fit isochrone is then superimposed on each CMD (Fig. 2). In this way updated estimates of the basic parameters (age, distance and reddening) are finally derived (see Table 6). Distances are computed adopting $A_{V}=3.1 \cdot E(B-V)$ (Moitinho 2001). Finally, by assuming $8.5 \mathrm{kpc}$ as the Sun's distance from the Galactic center, we provide in Table 7 the Cartesian Galactic coordinates $X_{\mathrm{GC}}, Y_{\mathrm{GC}}$ and $Z_{\mathrm{GC}}$, and the distance $\left(R_{\mathrm{GC}}\right)$ of the clusters from the Center of the Galaxy. The Cartesian coordinates are defined as $Z$ pointing toward the North Galactic Pole, $Y$ toward the direction of the Galactic rotation, and, finally, $X$ pointing toward the anti-center.

The fundamental parameters are thus summarized in Tables 5-7.

\subsection{Berkeley 75}

For this cluster we make use of the $B V I$ photometry from Carraro et al. (2005a). We have only two stars with spectroscopy and their velocities are clearly incompatible (see Table 2), as are their metallicities (Table 4). In Fig. 2 (panel a) we have indicated these two stars with squares. The decision on the possible membership of these stars is obviously quite difficult. In the figure we show as a member (filled square) the fainter (\#22) of the two stars, and suggest that star \#9 (open square) is actually an interloper. First, star \#22 falls closer to the adjusted isochrone in the CMD, while star \#9 seems too bright and blue. In addition, in the figure (where only the stars within the cluster radius are shown), while star \#22 lies within the cluster radius, star \#9 is close to the edge of the field of view, well outside the cluster radius; this star is shown in Fig. 2a only because it was observed spectroscopically. Finally, from Table 2 one can see how star \#9 is actually cooler than star \#22. If one derives their reddening from the intrinsic colors (Worthey et al. 2006), it turns out that star \#22 has a reddening compatible with the bulk of the MS stars (0.04), while star \#9 basically does not have reddening, being therefore a nearby giant star. The probable member (\#22) has a metallicity $[\mathrm{Fe} / \mathrm{H}]=-0.22 \pm 0.20$, and this translates into $Z=0.011$ (Carraro et al. 1999). A 4 Gyr isochrone for this metallicity has been super-imposed in Fig. 2a shifted by $E(B-V)=0.04$ and $(m-M)_{V}=14.9$. Note that our inferred distance of this cluster turns out to be lower than previous estimates (Table 1, Carraro et al. 2005a) due to the much higher metallicity we derived from spectroscopy. However, we consider our derived values for this cluster to be very preliminary since they are in part based on only one star with ambiguities in determining its membership.

\subsection{Berkeley 25}

The photometry for this cluster is also taken from Carraro et al. (2005a). We have five stars with very similar radial velocities, and therefore all of the measured stars are probable members. 

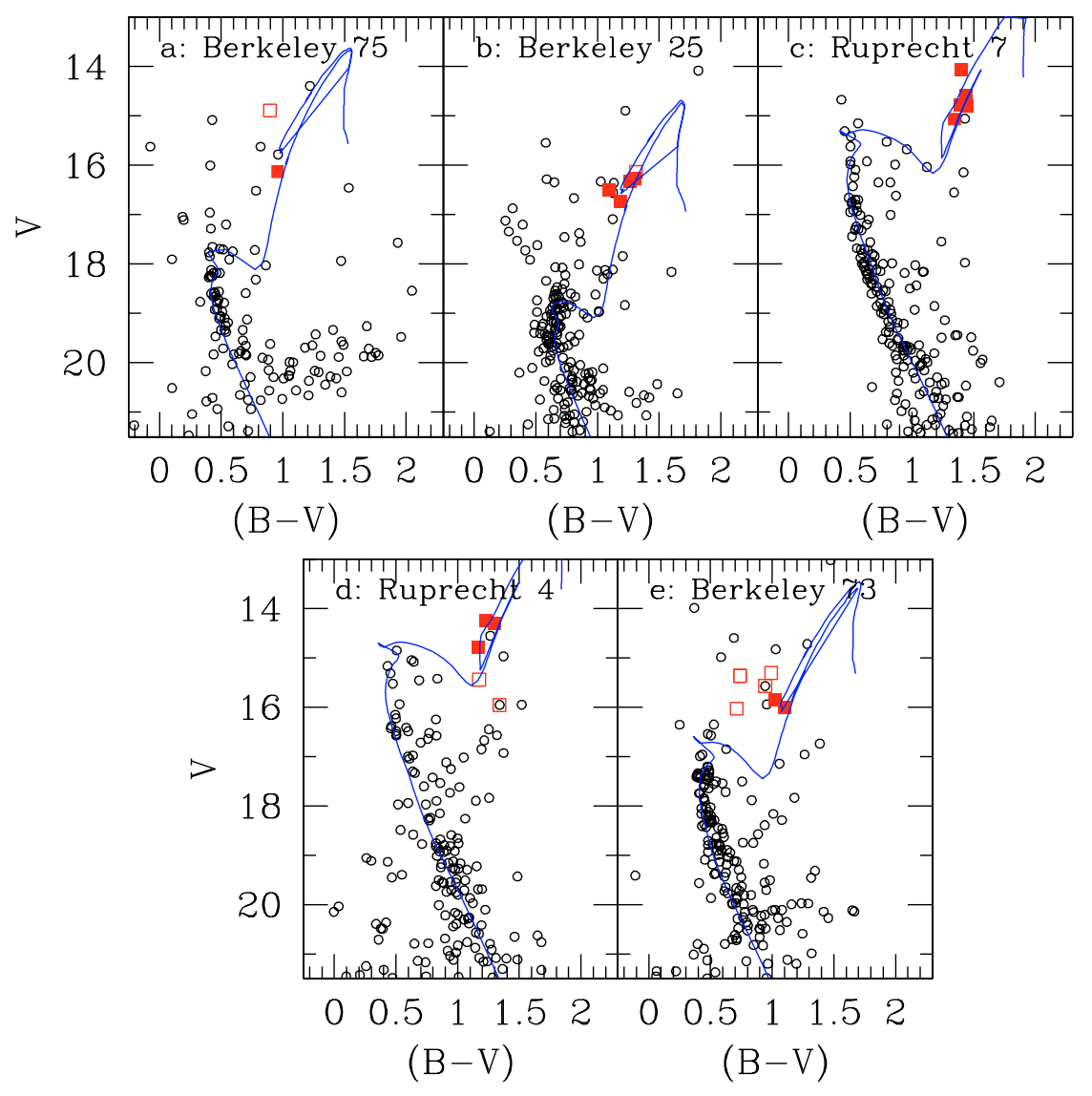

Fig. 2. Revision of clusters' fundamental parameters. In each panel we show the isochrone solutions and indicate radial velocity members (filled squares) and non-members (empty squares) for a) Berkeley 75 , b) Berkeley 25, c) Ruprecht 7 , d) Ruprecht 4, and e) Berkeley 73, respectively.

Table 5. Mean abundance analysis from cluster members. In parenthesis below the element the number of lines used is indicated.

\begin{tabular}{|c|c|c|c|c|c|c|c|c|c|c|}
\hline 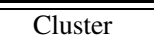 & $\overline{\overline{[\mathrm{Fe} / \mathrm{H}]}}$ & $\overline{\overline{[\mathrm{Mg} / \mathrm{H}]}}$ & $\overline{\overline{[\mathrm{Si} / \mathrm{H}]}}$ & $\overline{\overline{[\mathrm{Ca} / \mathrm{H}]}}$ & $\overline{\overline{[\mathrm{Ti} / \mathrm{H}]}}$ & 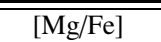 & $\overline{\overline{[\mathrm{Si} / \mathrm{Fe}]}}$ & $\overline{\overline{[\mathrm{Ca} / \mathrm{Fe}]}}$ & $\overline{\overline{[\mathrm{Ti} / \mathrm{Fe}]}}$ & $\overline{\overline{[\alpha / \mathrm{Fe}]}}$ \\
\hline & (150) & (3) & (13) & (16) & (33) & & & & & \\
\hline$\overline{\text { Ber }}$ & $.22 \pm 0.20$ & $+0.16 \pm$ & $42 \pm 0.14$ & $24 \pm 0.07$ & $06 \pm 0.04$ & $\overline{9}$ & 7 & 10 & & 0.08 \\
\hline & $20 \pm$ & 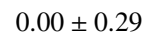 & & & 3 & & & 12 & & -0.01 \\
\hline Ruprecht 7 & $-0.26 \pm 0.05$ & $-0.35 \pm 0.09$ & $-0.20 \pm 0.05$ & $-0.22 \pm$ & $-0.37 \pm 0.09$ & -0.10 & $+0.05 \pm$ & $+0.03 \pm$ & $-0.12 \pm 0.10$ & -0.04 \\
\hline Ruprecht 4 & $-0.09 \pm 0.05$ & $-0.09 \pm 0.04$ & $-0.05 \pm 0.04$ & $-0.05 \pm 0.05$ & $-0.06 \pm 0.05$ & $+0.01 \pm 0.05$ & $+0.05 \pm 0.05$ & $+0.05 \pm 0.06$ & $+0.04 \pm 0.06$ & +0.04 \\
\hline Berkeley 73 & $-0.22 \pm 0.10$ & $-0.12 \pm 0.10$ & $-0.05 \pm 0.04$ & $-0.30 \pm 0.07$ & $-0.17 \pm 0.07$ & $+0.10 \pm 0.15$ & $+0.17 \pm 0.09$ & $-0.08 \pm 0.19$ & $+0.05 \pm 0.12$ & +0.06 \\
\hline
\end{tabular}

However, only for three of them (\#10, 12, and 13) could we perform detailed abundance analysis, while for the other two only an estimate of the iron abundance was possible, due to the lower $S / N$. These $[\mathrm{Fe} / \mathrm{H}]$ values are comparable within the error among all members, except for star \#10, which we are here considering as a probable non-member. These stars are indicated with filled squares in Fig. 2b. The mean of the four metallicity measures (Table 4) yields $[\mathrm{Fe} / \mathrm{H}]=-0.20 \pm 0.05$, which implies $Z=0.012$. The best-fit is for a $5 \mathrm{Gyr}$ isochrone, and it is shown in the same panel. It implies $E(B-V)=0.11$ and $(m-M)_{V}=15.6$.

\subsection{Ruprecht 7}

The photometry of this cluster comes from Carraro et al. (2005b). We obtained spectra for five stars (Table 2) and all have compatible radial velocities and metallicities within the errors. The photometry of these stars is indicated in Fig. 2c. The mean of the five metallicity measures yields $[\mathrm{Fe} / \mathrm{H}]=-0.26 \pm 0.05$, which corresponds to $Z=0.010$. The $800 \mathrm{Myr}$ isochrone that provides the best fit for this metallicity has been drawn for $E(B-V)=0.36$ and $(m-M)_{V}=15.0$. Owing to the lower metallicity we measure, these findings somewhat deviate from the purely photometric estimates by Carraro et al. (2005b).

\subsection{Ruprecht 4}

Of the five stars with Carraro et al. (2005b) photometry for which we obtained spectra, we propose that three (\# 3,4 and 8) are cluster members (filled squares in Fig. 2d), while the other two (the two faintest) are interlopers. This choice is also motivated by the better position of the candidate member stars in the CMD (see Fig. 2d). Four of the five stars lie near the cluster center (1.5 arcmin, Carraro et al. 2005b), but star \#29 is located well outside, suggesting it is a probable non-member. This further corroborates our member selection. From the three adopted members, we derive a mean $[\mathrm{Fe} / \mathrm{H}]=-0.09 \pm 0.05$, hence $Z=0.015$. The $800 \mathrm{Myr}$ isochrone for this metal content has been superimposed using $E(B-V)=0.30$ and $(m-M)_{V}=14.30$.

\subsection{Berkeley 73}

The photometry of this cluster comes from Carraro et al. (2005a). In this case assigning spectroscopically-based 
memberships is again quite a difficult task. Among the six stars for which we obtained UVES spectra, we do not consider the two stars with dwarf-like gravities, \#13 and 19, as members, while we consider the stars \#16 and 18 as members, because they are both giants with comparable velocities and metallicities. Star \#15 is a probable radial velocity non-member, as is star \#12. We therefore are left with two stars we consider to be members, and their mean $[\mathrm{Fe} / \mathrm{H}]$ is $-0.22 \pm 0.05$. This translates to $Z=0.011$, as in the case of Berkeley 75 . The $1.5 \mathrm{Gyr}$ best fit isochrone shown in Fig. 2e has been shifted by assuming $E(B-V)=0.11$ and $(m-M)_{V}=15.30$, a result consistent with the purely photometric analysis by Carraro et al. (2005a).

\section{Extension of the cluster sample}

Out of 5 total, 3 of our sample (the Berkeley clusters) are at heliocentric distances larger than $7 \mathrm{kpc}$, and therefore have to be carefully considered as possible previously neglected MRi candidates (Crane et al. 2003; Frinchaboy et al. 2004). Therefore they deserve further attention to better describe this tidal feature. At the same time, we want to investigate the chemical and kinematic properties of the outer Galactic disk using old open clusters.

To these aims, we enlarge the sample presented in the previous Sections by adding eight additional distant anti-center clusters for which high resolution abundances are available. These clusters are: Berkeley 29 and Saurer 1 (Carraro et al. 2004), Berkeley 22 (Villanova et al. 2005), Tombaugh 2 (Frinchaboy et al. 2007), Berkeley 21 (Hill \& Pasquini 1999; Yong et al. 2005), and Berkeley 20, Berkeley 31, and NGC 2141 from Yong et al. (2005). These additional clusters are all located in the Third Galactic Quadrant, having $187^{\circ} \leq l \leq 240^{\circ}$ and $-18^{\circ} \leq b \leq$ $+8^{\circ}$. Their properties are summarized in Table 8 . These thirteen clusters all lie more than $12 \mathrm{kpc}$ from the Galactic center and represent the largest sample of anti-center distant clusters with reliable chemical abundance measurements analyzed together so far. By the way, 3 of them (Berkeley 29, Saurer 1 and Tombaugh 2) have been previously suggested to be MRi candidates.

In order to put all the cluster $[\mathrm{Fe} / \mathrm{H}]$ measurements taken from the literature on the same metallicity scale as the present study clusters, we used the values for Berkeley 29, which is in common between Yong et al. (2005) and Carraro et al. (2004). This allows us to scale Berkeley 20, Berkeley 31 and NGC 2141, and Berkeley 21. For this latter we still consider the Yong et al. value, since it is basically identical to Hill \& Pasquini (1999) estimate. For Berkeley 29, Yong et al. report an $[\mathrm{Fe} / \mathrm{H}]$ value 0.1 dex lower than Carraro et al. (2004). Therefore we increase all Yong et al. $[\mathrm{Fe} / \mathrm{H}]$ values by $0.1 \mathrm{dex}$.

\section{Abundance ratios}

Carraro et al. (2004) and Yong et al. (2005) have emphasized that the few outer Galactic disk clusters insofar studied show a sizeable enhancement in $\alpha$ elements over the solar ratio. Having a larger sample, we investigate here what the $\alpha$ element ratios are suggesting to us about the origin of these clusters, say whether they are genuine disk clusters, or whether they are the signature of an extra-galactic population. Indeed one may speculate that an $\alpha$ element over-abundance indicates that the material in the outer disk formed rapidly, possibly due to a relatively recent merging event (2-5 Gyr ago). This seems to be confirmed by the ages of the clusters under analysis. None of the clusters in our sample is very old (ages less than 5 Gyr), which may suggest that very old open clusters (like NGG 6791, Berkeley 17, Collinder 261 and NGC 188 to name a few examples) do not populate the anti-center. We emphasize however that this might be the result of a selection effect, which further studies have to clarify. Nonetheless, if this age distribution is real, one may speculate that these clusters could have formed during a merger, or deposited by a merger. In fact, according to our current understanding of disk formation, we expect that the outer parts have been forming later (inside out scenario) and that the more recently accreted material, if any, is around the outer edge of the disk.

We could measure several $\alpha$ elements for our five clusters, as listed in Table 5. Figure 3 shows the $[\mathrm{Fe} / \mathrm{H}]$ trends of four abundance ratios: $[\mathrm{Mg} / \mathrm{Fe}],[\mathrm{Si} / \mathrm{Fe}],[\mathrm{Ca} / \mathrm{Fe}]$, and $[\mathrm{Ti} / \mathrm{Fe}]$ of our 5 program clusters, plus the 8 additional clusters collected from the literature. The dashed lines are linear fits through the data points with slopes of $-0.14 \pm 0.11,-0.58 \pm 0.19,-0.28 \pm 0.12$, and $-0.08 \pm 0.10$ for $[\mathrm{Mg} / \mathrm{Fe}],[\mathrm{Si} / \mathrm{Fe}],[\mathrm{Ca} / \mathrm{Fe}]$, and $[\mathrm{Ti} / \mathrm{Fe}]$, respectively. There is a trend of having decreasing $\alpha$ elements ratios at increasing metallicity $([\mathrm{Fe} / \mathrm{H}])$ for all the measured elements, except for Ti.

Inspecting this figure we notice that these clusters define a trend of $\alpha$ element abundances versus $[\mathrm{Fe} / \mathrm{H}]$ consistent with Galactic open clusters (Friel et al. 2003, Fig. 5) and field F and G stars (Reddy et al. 2003, Fig. 8) near the Sun in the same metallicity range of our sample. The generally enhanced abundances are more reminiscent of the thick than thin disk behavior (Reddy et al. 2003).

At these metallicities there are no dwarf galaxies having the same abundance ratios. The Sagittarius Dwarf Spheroidal does reach these high metallicities $(-0.5 \leq[\mathrm{Fe} / \mathrm{H}] \leq 0.0)$, however its stars tend to be significantly under-abundant in $\alpha$ elements. The unpublished work by Smecker-Hane \& McWilliam (astro-ph/0205411) present a few determinations compatible with our clusters. However, more recent studies (Sbordone et al. 2007, and references therein) based on a larger sample show that in this metallicity range $\alpha$ elements are significantly sub-solar.

In conclusion, the $\alpha$ element ratios of outer disk open clusters are more compatible with the solar vicinity thin/thick disk than with dwarf Galaxies, suggesting that these clusters are genuine members of the Galactic disk.

\section{Age-metallicity relationship}

The age-metallicity relationship (AMR) is another important diagnostic of galaxy formation scenarios. If our clusters were born in a distinct galaxy, they would likely show an AMR distinct from the clusters intrinsic to our Galaxy.

For example, Frinchaboy et al. (2004) suggest the open and globular clusters putatively associated with MRi show an AMR similar to that of the Sagittarius dwarf galaxy (Layden \& Sarajedini 2000). Forbes et al. (2004) built on this concept and used the AMR as a tool to decide the membership or not of potential CMa/MRi clusters, as did Frinchaboy et al. (2006). However, both these studies are based on highly inhomogeneous samples, combine together open and globular clusters, and make use of photometry- or low-resolution-sprectroscopy-based metallicities.

It is quite well accepted that the bulk of the old open clusters in the disk do not define a clear-cut AMR (Friel 1995; Carraro et al. 1998; Friel et al. 2002), but rather at any age these clusters show a large scatter in metallicity. Typically, at any age 
Table 6. Revision of the fundamental parameters of the clusters under study.

\begin{tabular}{ccccccc}
\hline \hline Name & {$[\mathrm{Fe} / \mathrm{H}]$} & Metallicity & $E(B-V)$ & $(m-M)$ & $d_{\odot}$ & Age \\
\hline & $\mathrm{dex}$ & & & & $\mathrm{kpc}$ & $\mathrm{Gyr}$ \\
\hline Berkeley 75 & $-0.22 \pm 0.20$ & 0.011 & $0.04 \pm 0.03$ & $14.90 \pm 0.20$ & 9.10 & $4.0 \pm 0.4$ \\
Berkeley 25 & $-0.20 \pm 0.05$ & 0.012 & $0.11 \pm 0.05$ & $15.60 \pm 0.30$ & 11.40 & $5.0 \pm 0.5$ \\
Ruprecht 7 & $-0.26 \pm 0.05$ & 0.010 & $0.36 \pm 0.05$ & $15.00 \pm 0.20$ & 6.00 & $0.8 \pm 0.2$ \\
Ruprecht 4 & $-0.09 \pm 0.05$ & 0.015 & $0.30 \pm 0.05$ & $14.30 \pm 0.20$ & 4.70 & $0.8 \pm 0.2$ \\
Berkeley 73 & $-0.22 \pm 0.10$ & 0.011 & $0.11 \pm 0.05$ & $15.30 \pm 0.20$ & 9.80 & $1.5 \pm 0.3$ \\
\hline
\end{tabular}

Table 7. Revision of the fundamental parameters of the clusters under study.

\begin{tabular}{ccccccccccc}
\hline \hline Name & $l$ & $b$ & {$[\mathrm{Fe} / \mathrm{H}]$} & $X$ & $Y$ & $Z$ & $R_{\mathrm{GC}}$ & $R V$ & $V_{\mathrm{GSR}}$ & $V_{\mathrm{ROT}}$ \\
\hline & {$[\mathrm{deg}]$} & {$[\mathrm{deg}]$} & $\mathrm{dex}$ & $\mathrm{kpc}$ & $\mathrm{kpc}$ & $\mathrm{kpc}$ & $\mathrm{kpc}$ & $\mathrm{km} \mathrm{s}^{-1}$ & $\mathrm{~km} \mathrm{~s}^{-1}$ & $\mathrm{~km} \mathrm{~s}^{-1}$ \\
\hline Berkeley 75 & 234.30 & -11.12 & $-0.22 \pm 0.20$ & 13.7 & -7.2 & -1.6 & 15.5 & $+94.6 \pm 0.35$ & -95.7 & 220.5 \\
Berkeley 25 & 226.60 & -9.69 & $-0.20 \pm 0.05$ & 16.2 & -8.1 & -1.9 & 18.2 & $+134.3 \pm 0.20$ & -39.1 & 117.4 \\
Ruprecht 7 & 225.44 & -4.58 & $-0.26 \pm 0.05$ & 12.7 & -4.6 & -0.5 & 13.5 & $+76.6 \pm 0.50$ & -95.0 & 210.9 \\
Ruprecht 4 & 222.04 & -5.31 & $-0.09 \pm 0.05$ & 12.0 & -3.1 & -0.4 & 12.4 & $+47.5 \pm 1.00$ & -114.5 & 250.2 \\
Berkeley 73 & 215.28 & -9.42 & $-0.22 \pm 0.10$ & 16.4 & -5.6 & -1.6 & 17.4 & $+95.7 \pm 0.20$ & -44.9 & 155.0 \\
\hline
\end{tabular}

between 1 and $8 \mathrm{Gyr}$, metallicity ranges from $[\mathrm{Fe} / \mathrm{H}] \sim-0.5$ to $\mathrm{Fe} / \mathrm{H}] \sim+0.05$ (Friel et al. 2002, Fig. 4).

This seems to be the case also for outer disk clusters (see Fig. 4): At all ages from 0.8 to 5 Gyr there is a sizable spread in $[\mathrm{Fe} / \mathrm{H}]$ extending up to $0.4 \mathrm{dex}$, in good agreement with the trend of old open clusters in the solar neighborhood. The two lines in Fig. 4 show the full metallicity range that old open clusters in the disk exhibit at any age (Friel et al. 2002).

There might be a possible hint of an AMR if we remove the two most metal-poor clusters (Berkeley 21 and NGC 2141, Yong et al. 2005) located at about 2 Gyr. However, despite the large errors, the metallicity of these two clusters are consistent with all the determinations obtained so far (Hill \& Pasquini 1999), and there are no reason to believe they are wrong.

Therefore, the analysis of the AMR lends further support to the idea that the outer disk old open clusters do not differ from typical disk clusters, since they do show the same lack of any AMR as typical disk open clusters and argue against their origin in a putative dwarf galaxy.

\section{Metallicity distribution}

The sample of clusters we are analyzing has a mean metallicity $[\mathrm{Fe} / \mathrm{H}]=-0.34 \pm 0.15$. It is useful to compare this value with the available measures for the MRi. Yanny et al. (2003) derived $[\mathrm{Fe} / \mathrm{H}]=-1.6 \pm 0.3$ from $\mathrm{Ca}$ II $(\mathrm{K})$ line strenghts of MS blue $\mathrm{F} / \mathrm{G}$ star spectra. This metallicity has been recently confirmed by Wilhelm et al. (2005), who used synthetic spectra and colors to derive $[\mathrm{Fe} / \mathrm{H}]=-1.37 \pm 0.04$ from a group of $\mathrm{A} / \mathrm{F}$ stars. From the spectra of a sample of $M$ giants Crane et al. (2003) obtained quite a different result. The Ca II $\lambda \lambda 8498,8542$ and $\mathrm{Mg}$ I $\lambda 8807$ lines synthesis yielded $[\mathrm{Fe} / \mathrm{H}]=-0.4 \pm 0.3$. If the $\mathrm{F} / \mathrm{G}$ MS stars and the M giants belong to the same population, they seem to define an AMR, provided they are sampling different age sub-populations, corresponding to different rounds of star formation. If, on the contrary, $\mathrm{F} / \mathrm{G}$ and $\mathrm{M}$ stars do belong to the same population and possess roughly the same age, clearly something is wrong with these estimates of the metallicity.

Interestingly, our clusters do have the same metallicity as the Crane et al. (2003) M giants. One may be tempted to associate old open clusters with $\mathrm{M}$ giants, while associating putative members globular clusters (Frinchaboy et al. 2004) with F/G stars. While in principle this might be correct - the Sgr DSph

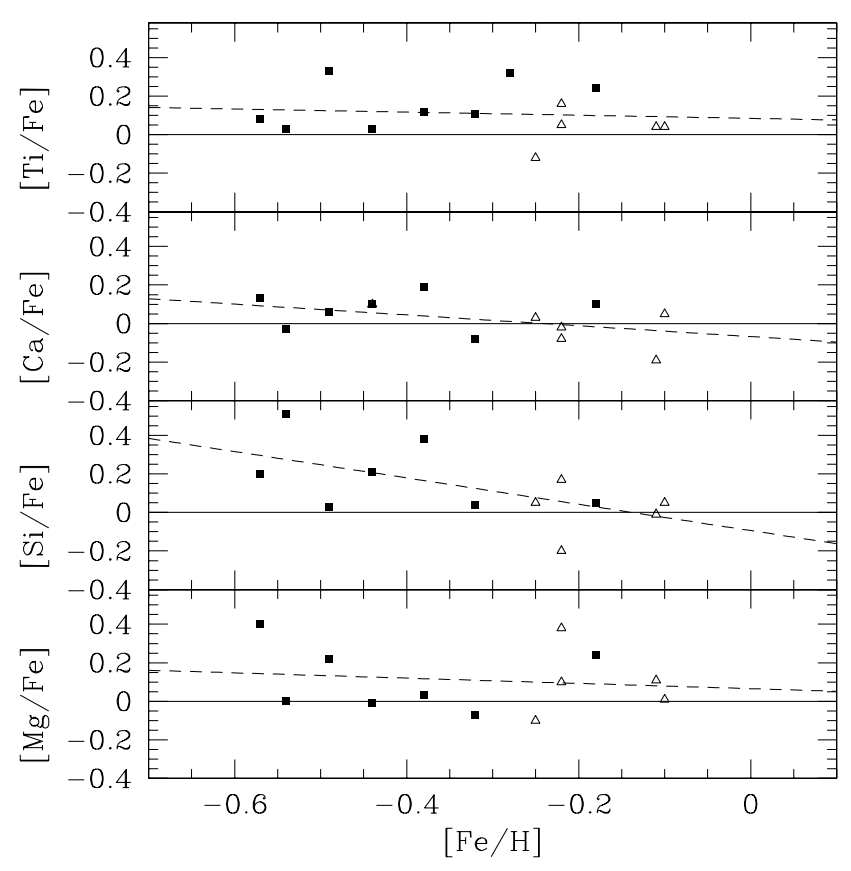

Fig. 3. The trend of $\alpha$ element abundances with metallicity. Open triangles indicate the five program clusters, while filled squares are additional anti-center clusters culled from the literature.The dashed line is a fit through the data.

represents a clear counterpart - such a conclusion still remains premature, first because $\alpha$ elements and AMR are suggesting a different scenario, second because the MRi global properties are still very poorly known.

Besides, one is left with the question as to why outer disk star clusters should have roughly the same metallicity distribution as solar vicinity star clusters (Friel et al. 2002).

\section{The radial abundance gradient in the outer Galactic disk}

The most recent determination of the slope of the radial abundance gradient using old open clusters in the Galactic disk is presented in Friel et al. (2002). From a sample of about 40 


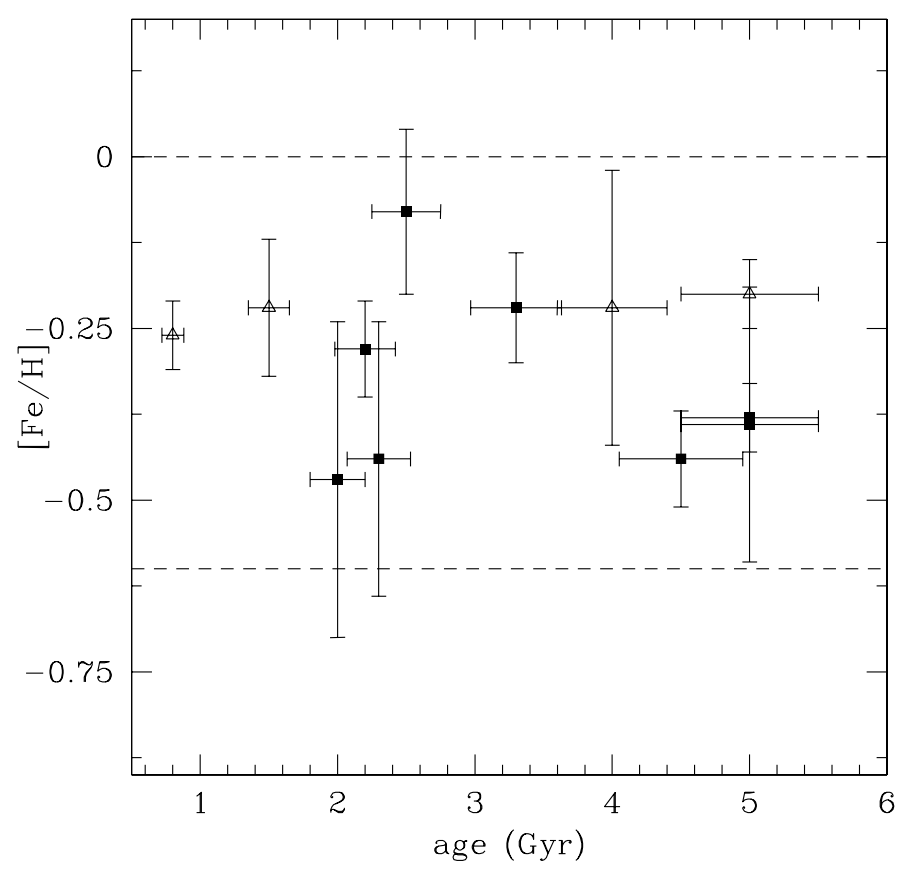

Fig. 4. Age metallicity distribution, with symbols as in Fig. 3. The dashed lines indicate the metallicity range of old open clusters in the Galactic disk, from Friel et al. (2002).

clusters located between 8 and $14 \mathrm{kpc}$ from the Galactic center a mean slope of $-0.06 \mathrm{dex} \mathrm{kpc}^{-1}$ is found. This slope mostly represents the abundance gradient in the solar vicinity, since the sample contains just one cluster beyond $14 \mathrm{kpc}$ from the center of the Milky Way and only five clusters outside of $12 \mathrm{kpc}$.

Carraro et al. (2004) and Yong et al. (2005) provide evidence that the abundances in the outer disk $\left(R_{\mathrm{GC}}\right.$ larger than $12-13 \mathrm{kpc}$ ) do not follow the expectations of the extrapolation of the gradient as determined by clusters in the solar neighborhood, but instead the metallicity gradient seems to flatten at $R_{\mathrm{GC}} \sim 12-14 \mathrm{kpc}$.

With the supplement of the eight additional clusters from the literature we now have a unique sample of clusters to reassess the radial abundance gradient in the outskirts of the Galactic disk. Our sample spans almost $10 \mathrm{kpc}$ in Galactocentric distance (see Table 5 and 8 ), from $R_{\mathrm{GC}}=12.0$ to $21.6 \mathrm{kpc}$. Moreover, they are all located essentially toward the same Galactic region $\left(186^{\circ} \leq l \leq 234^{\circ}\right)$. Figure 5 plots the metallicity trend of all of the aforementioned clusters together with their metallicity uncertainty.

In this figure, the solid line is the radial abundance gradient from Friel et al. (2002) sample. Clearly, distant anticenter old clusters deviate from this trend, and show how the mean gradient beyond $12 \mathrm{kpc}$ flattens out and essentially maintains a constant value of $[\mathrm{Fe} / \mathrm{H}] \sim-0.35$.

If we consider the Friel et al. (2002) clusters and the ones discussed here together, a global gradient of $-0.018 \pm$ $0.021 \mathrm{dex} \mathrm{kpc}^{-1}$ results (dashed line) - much flatter than that found by Friel et al. (2002) and compatible with the Galactic disk not having any gradient when using old open clusters over its full extent.

We note that shallow metallicity gradients across the full span of spiral galaxy disks are commonly found (see Moustakas \& Kennicut 2006, and references therein).

The global value of the gradient we find is also consistent with that predicted by Cescutti et al. (2006, see below) chemical evolution models, which yield a gradient of $\sim-0.05 \mathrm{dex} \mathrm{kpc}^{-1}$ all the way from 4 to $22 \mathrm{kpc}$ from the Galactic center.

In Fig. 6 we show the radial abundance gradient of four $\alpha$-elements $-[\mathrm{Mg} / \mathrm{H}],[\mathrm{Si} / \mathrm{H}],[\mathrm{Ca} / \mathrm{H}]$, and $[\mathrm{Ti} / \mathrm{H}]-$ and derive their gradient slope (indicated on the right side of each panel).

Recently, Cescutti et al. (2006) calculated the slopes of the Galactic abundance gradient of several elements as derived from chemical evolution models of the Milky Way. This model is based on the assumption that the MW evolved basically in isolation, but with different star formation thresholds imposed for the halo and disk. The disk is modeled as an inside-out growing structure. Modern prescriptions for element production rates are employed.

In the same Galactocentric distance baseline (12 to $22 \mathrm{kpc}$ ) the Cescutti et al. models yield abundance gradient slopes of about $-0.020 \mathrm{dex} \mathrm{kpc}^{-1}$ for the four above elements, while reproducing at the same time the observed gradient in the solar vicinity.

Therefore, model predictions are consistent with our new results within the errors, except for $[\mathrm{Si} / \mathrm{H}]$, for which we found a shallower slope than the models, consistent with no gradient. We find that the mean trend of the models match the trend of the observations for the outer disk.

\section{Spatial distribution and kinematics}

The five program clusters are all located below the nominal Galactic plane and reach as much as $2 \mathrm{kpc}$ below the plane (see Table 7). In Fig. 7 we plot the $R_{\mathrm{GC}}-Z_{\mathrm{GC}}$ distribution of all thirteen clusters beyond $12 \mathrm{kpc}$ from the Galactic Center collected in Sect. 5. In the third Galactic quadrant, stars and clusters preferentially lie below the formal $b=0$ plane of the Galaxy, following the pattern of the Galactic warp and flare (Moitinho et al. 2006). These are shown for different values of $b$, from $0^{\circ}$ (solid line) to $\pm 3^{\circ}$ (dashed lines) from Momany et al. 2006, which the reader is referred to for any additional details. Berkeley 29, Berkeley 31 and Saurer 1 are at odds with the bulk of the sample and are located well above the Galactic plane. Still, they do not deviate significantly from the predictions of a warped/flared disk.

Scott et al. (1995) have studied the kinematics of old open clusters in the solar vicinity. From a sample of 35 clusters at mean Galactocentric distance of $10.3 \mathrm{kpc}$, they found a mean rotational velocity of $211 \pm 7 \mathrm{~km} \mathrm{~s}^{-1}$, consistent with the Galaxy rotation curve. Our sample contains clusters which are located much further away, and at higher Galactic latitudes. We therefore expect to find deviations from purely circular motions. Although these deviations might be important signatures of accretion, one has to be very carefully analysing distant star clusters in the anticenter, since both their radial velocity is generally dominated by non circular motions and the disk structure is more complicated than in the solar neighborhood.

Anyhow, we derive circular velocities for these clusters, using Olling \& Merrifield's (2000) Eqs. (1) and (2), which combined together yield:

$V_{\mathrm{ROT}}=\frac{R_{\mathrm{GC}}}{8.5} \cdot\left[220+\frac{R V}{\sin l \cos b}\right]$,

where the circular speed and distance from the Galactic Center of the Sun are taken to be $220 \mathrm{~km} \mathrm{~s}^{-1}$ and $8.5 \mathrm{kpc}$, for consistency with the adopted values to derive cluster Galactic coordinates (see Sect. 5). Finally, RV here represents the Local Standard of Rest (LSR) corrected radial velocity.

Figure 8 shows the derived rotational velocity distribution of the clusters as a function of Galactocentric radius, compared 
Table 8. Additional clusters gathered from the literature. References are: (a) Carraro et al. (2004); (b) Yong et al. (2005); (c) Villanova et al. (2005); (d) Frinchaboy et al. (2007).

\begin{tabular}{|c|c|c|c|c|c|c|c|c|c|c|c|c|c|c|}
\hline Name & $\overline{l l}$ & $\bar{b}$ & $\bar{R} R V_{\mathrm{H}}$ & $\overline{d_{\odot}}$ & $R_{\mathrm{GC}}$ & $\overline{\overline{V_{\mathrm{ROT}}}}$ & 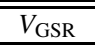 & {$[\mathrm{Fe} / \mathrm{H}]$} & 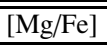 & [Si/Fe] & {$[\mathrm{Ca} / \mathrm{Fe}]$} & {$[\mathrm{TTi} / \mathrm{Fe}]$} & age & Ref. \\
\hline & $\operatorname{deg}$ & $\operatorname{deg}$ & $\mathrm{km} \mathrm{s}^{-1}$ & $\mathrm{kpc}$ & $\mathrm{kpc}$ & $\mathrm{km} \mathrm{s}^{-1}$ & $\mathrm{~km} \mathrm{~s}^{-1}$ & $\operatorname{dex}$ & $\operatorname{dex}$ & dex & $\operatorname{dex}$ & dex & Gyr & \\
\hline Berkeley 29 & 197.98 & +8.05 & +24.6 & 13.2 & 21.6 & 340.0 & $\begin{array}{l}-51.1 \\
\end{array}$ & -0.44 & -0.01 & +0.21 & +0.10 & +0.03 & 4.5 & a) \\
\hline Saurer 1 & 214.68 & +7.38 & +104.6 & 13.2 & 19.2 & 141.6 & -32.7 & -0.38 & +0.05 & +0.38 & +0.19 & +0.12 & 5.0 & a) \\
\hline Berkeley 31 & 206.25 & +5.12 & +55.7 & 8.3 & 16.3 & 210.9 & -48.6 & -0.57 & +0.40 & +0.20 & +0.13 & +0.08 & 2.0 & b) \\
\hline Berkeley 22 & 199.88 & -8.08 & +95.3 & 6.0 & 14.3 & 43.3 & +7.8 & -0.32 & -0.07 & +0.04 & -0.08 & +0.11 & 3.3 & c) \\
\hline Berkeley 20 & 203.48 & -17.37 & +78.9 & 8.4 & 16.0 & 99.7 & -19.8 & -0.49 & +0.22 & +0.03 & +0.06 & +0.33 & 5.0 & b) \\
\hline Tombaugh 2 & 232.00 & -6.88 & +121.0 & 8.3 & 15.1 & 149.6 & -75.8 & -0.28 & & & & +0.32 & 2.2 & d) \\
\hline Berkeley 21 & 186.84 & -2.51 & +12.6 & 5.0 & 13.5 & 323.0 & -24.2 & -0.54 & +0.00 & +0.51 & -0.03 & +0.03 & 2.3 & b) \\
\hline NGC 2141 & 198.08 & -5.78 & +24.1 & 4.3 & 12.0 & 177.8 & -37.7 & -0.18 & +0.24 & +0.05 & +0.10 & +0.24 & 2.5 & b) \\
\hline
\end{tabular}

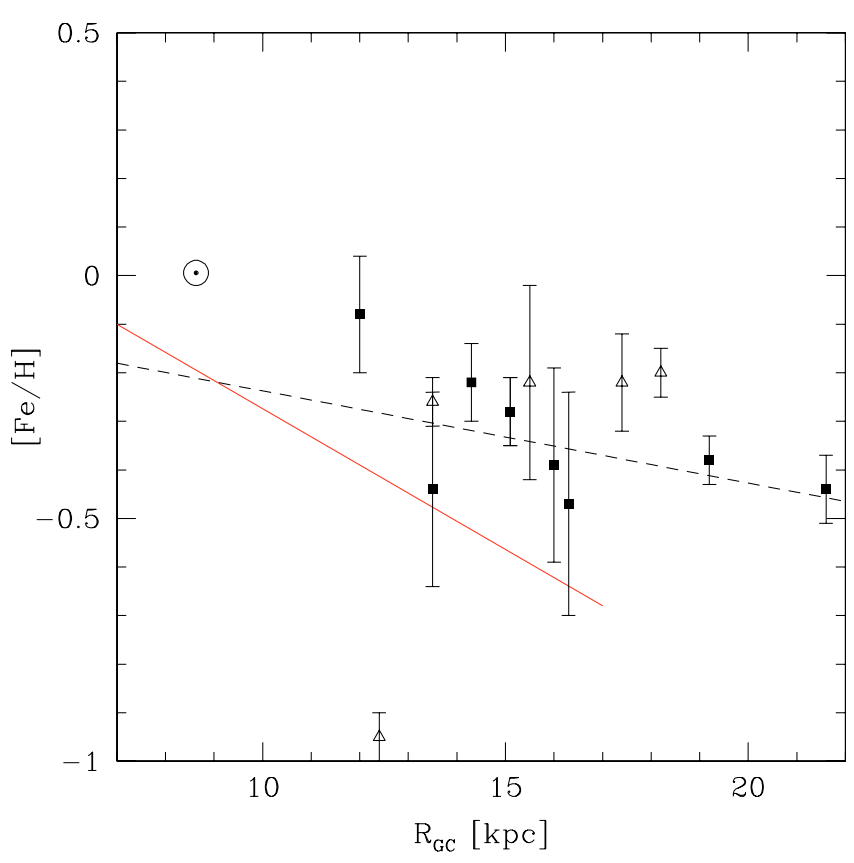

Fig. 5. The radial abundance gradient from old open clusters in the distant anticenter direction. Symbols are as in Fig. 3. The solid line is the Friel et al. (2002) mean abundance gradient, while the dashed line is the gradient when outer disk clusters are added.

to the Galaxy rotation curve from Olling \& Merrifield (2000; dashed line). The position of the Sun is indicated at $(8.5,200)$, while the range of values derived by Scott et al. (1995) for old open clusters is represented by the thick horizontal segment.

All the clusters rotate slower than predicted by the rotation curve, apart from Berkeley 29 and Berkeley 21, which have a significantly larger rotation velocity, but lie very close to the Galactic anticenter. Besides, the deviation from the Galaxy rotation curve increases with distance.

The mean rotational velocity of our clusters is about $150 \mathrm{~km} \mathrm{~s}^{-1}$, which differs from that of old open clusters in the solar vicinity (Scott et al. 1995), which rotate at the same speed as the Sun.

Before deriving conclusions on the meaning of this deviation, it is worth keeping in mind that these clusters are on the average much farther from the Galactic plane than the solar vicinity clusters, and their kinematics is more complex, possibly due to the warping and flaring of the disk.

To fully explore their kinematics we would need proper motion measurements, to derive cluster orbits and eccentricities

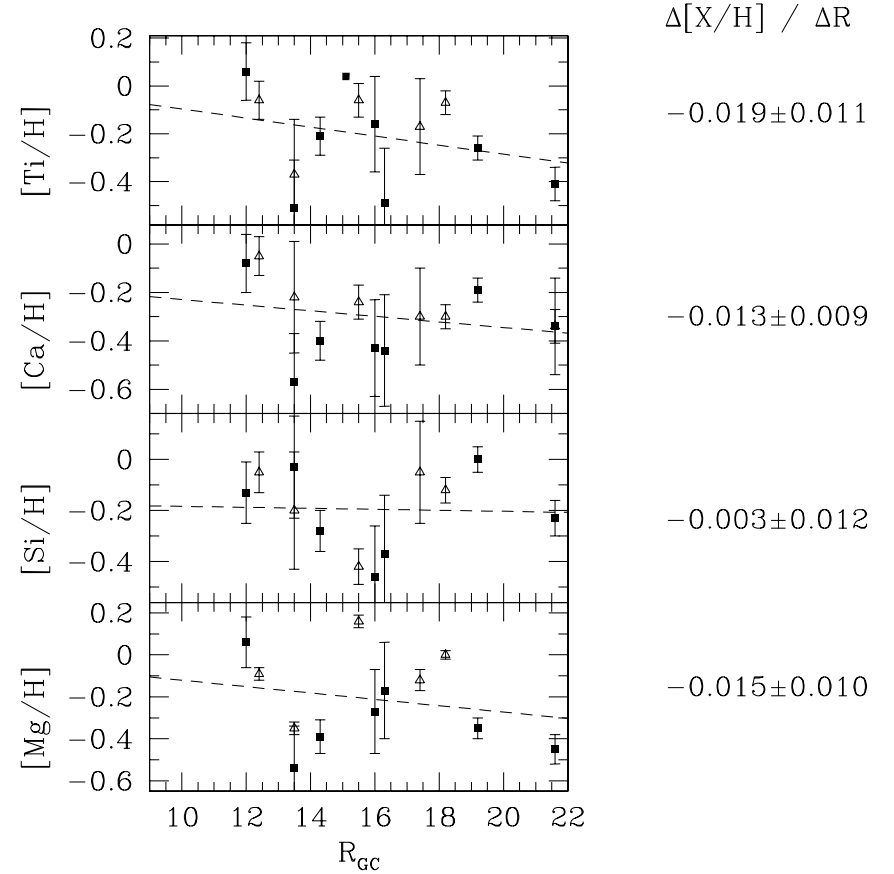

Fig. 6. The radial abundance gradient of $\mathrm{Mg}, \mathrm{Si}, \mathrm{Ca}$ and $\mathrm{Ti}$ from old open clusters in the distant Galactic anti-center. The symbols are the same as those used in Fig. 3. On the right the gradient slopes in the range 12 to $22 \mathrm{kpc}$ are indicated.

(Carraro \& Chiosi 1994; Frinchaboy 2006; Casetti-Dinescu et al. 2007). These quantities are mandatory to address the issue of the origin of these clusters, and hopefully disentangle between an extra-galactic origin, or the kinematic influence by a flared/warped disk.

At odd with the results from the chemical analysis, the study of kinematics presented here cannot help much to put more constraints on the formation, origin and evolution of the outer disk old open clusters system.

\section{Kinematic association to MRi}

The Galactic Anticenter Stellar Structure (GASS), or MRi, exhibits quite a tight relation between the velocity corrected to the Galactic standard of rest and the longitude in a sample of putative $M$ giant members (Crane et al. 2003). Based on that, Frinchaboy et al. $(2004,2006)$ looked for star clusters associated with this structure, and actually found that seven clusters (both open and globulars) are consistent with GASS membership 


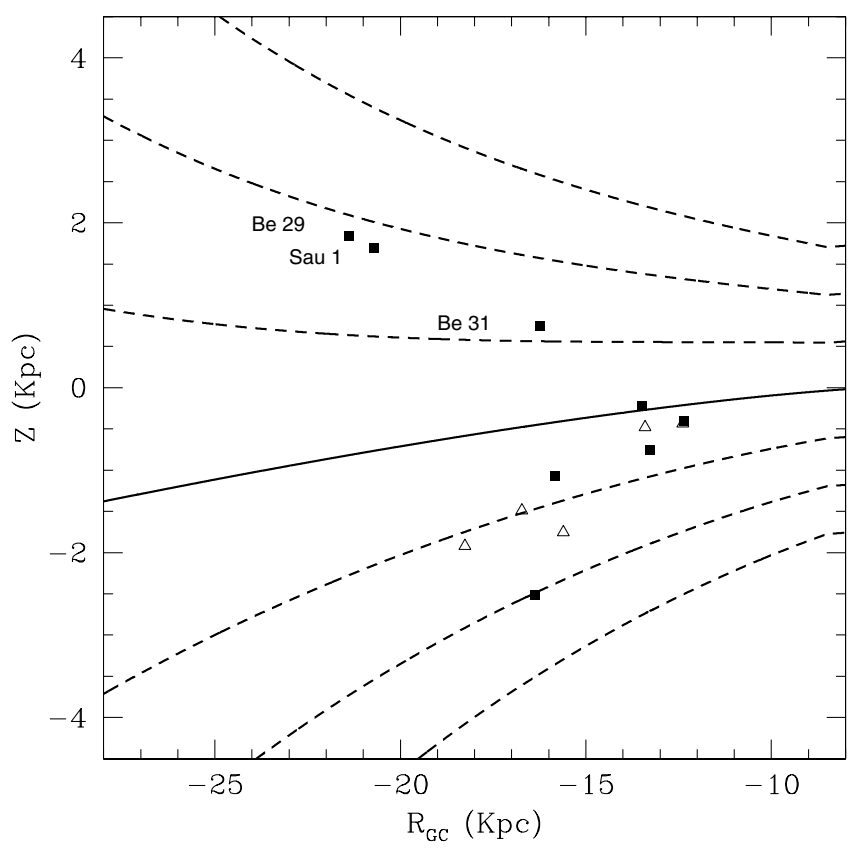

Fig. 7. Distribution of star clusters according to their height above the Galactic plane and distance from the Galactic center. The various lines indicate the warping/flaring of the disk for $b=0^{\circ}$ (solid line) and $\pm 1,2$ and 3 scale heights (dashed lines). Symbols are as in Fig. 3.

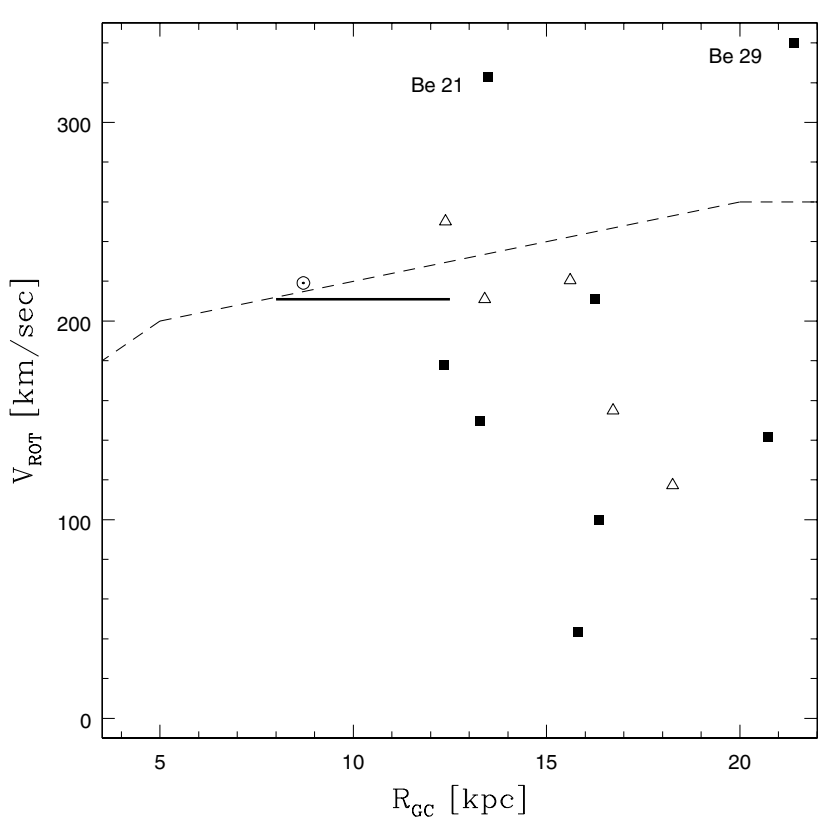

Fig. 8. Rotational velocity of old open clusters in the third Galactic quadrant beyond $12 \mathrm{kpc}$ from the Galactic center. The dashed line is the stellar disk rotation curve from Olling \& Merrifield (2000), while the thick solid line is the typical location of old open clusters in the solar vicinity. Symbols are as in Fig. 3.

in the Galactic standard of rest velocity $\left(v_{\mathrm{GSR}}\right)$ versus longitude plane.

Meanwhile, new radial velocities have been acquired for some of these clusters and others which are located in the MRi

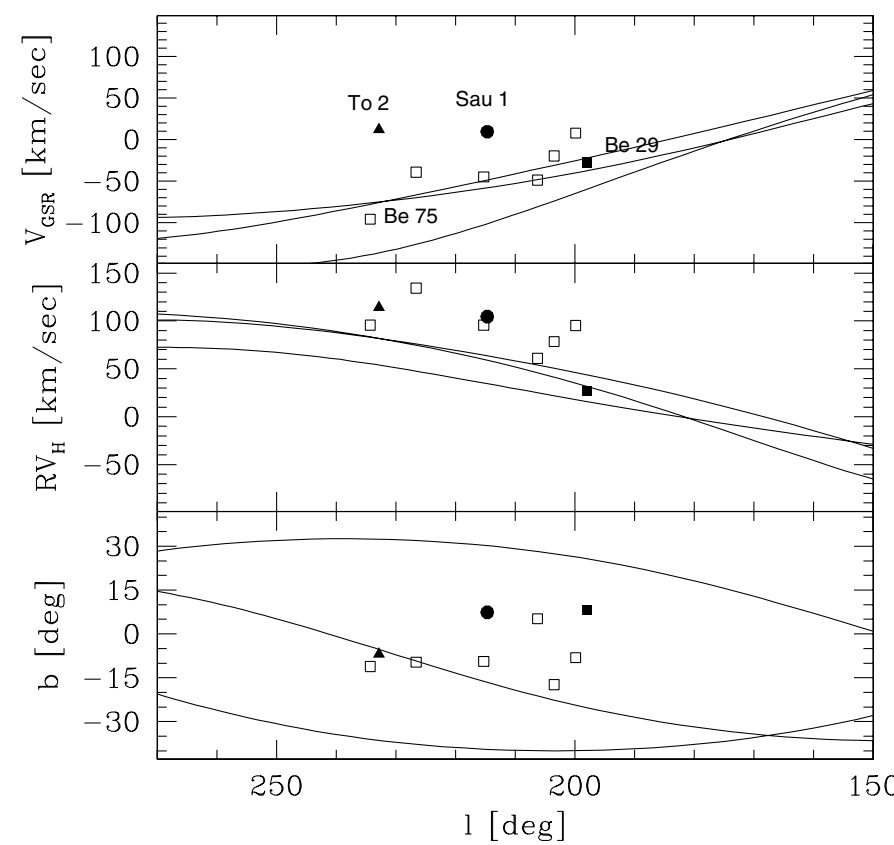

Fig. 9. Location and kinematics of old open clusters in the Galactic anti-center. Superimposed are Peñarubia et al. (2005) models of the Monoceros Ring. The filled circle indicates Saurer 1, whilst the filled square Berkeley 29 and the filled triangle Tombaugh 2, previously indicated as MRi members.

region. This permit us to revise the possible kinematic association of old open clusters with GASS.

In fact, Frinchaboy et al. 2006 could provide evidence that Saurer 1 and Berkeley 29 are probably associated with GASS.

We can here extend that analysis to the larger anti-center cluster sample we have described in previous Sections. To this aim, we computed $v_{\mathrm{GSR}}$ of each one of the five program clusters (see Table 7), and for the additional 8 clusters (Table 8).

The velocity $V_{\mathrm{GSR}}$ has been computed as:

$V_{\mathrm{GSR}}=R V_{H}+9 \cdot \cos (b) \cos (l)+(220+12) \cdot \cos (b) \sin (l)+7 \cdot \sin (b)(3)$

where $(9,12,7)$ are the components of the Sun peculiar motion - consistent with M giants from Majewski et al. (2003)-, and 220 is the Local Standard of Rest rotational velocity (see also Frinchaboy et al. 2006, Eq. (4)).

The aim is to compare cluster position and kinematics with the expected trends for the MRi, following the same approach as in Frinchaboy et al. (2006). Theoretical $-N$-body and analytical - calculations by Peñarrubia et al. (2005) are available that predict the location and kinematics of the MRi as a function of the shape of the Galactic halo.

Figure 9 compares the location and kinematics of old anticenter open clusters having heliocentric distances larger than $7 \mathrm{kpc}$ (small open squares), Berkeley 29, Tombaugh 2 and Saurer 1 (MRi candidates, filled square, triangle and circle, respectively) to the MRi models. This cluster sample, the largest so far of distant anti-center star clusters -including Berkeley 29 and Saurer 1- does not generally follow the expected MRi spatial distribution (bottom panel), while the heliocentric radial velocities (middle panel) are only marginally compatible with MRi models, as are the Galactic standard of rest velocities $v_{\mathrm{GSR}}$ (top panel).

Finally, we notice that while Berkeley 29 seems to follow the MRi models kinematics, but not the spatial location, Saurer 1 clearly deviates in all the diagrams, and Tombaugh 2 is only 
spatially compatible with MRi (but see Frinchaboy et al. 2007). Berkeley 75 at $l=234^{\circ}$ seems to be the cluster that simultaneously best matches the model expectations in the three planes.

This leads us to our final argument, now based on kinematics, indicating that the preferred origin of our clusters is Galactic as opposed to extra-galactic.

\section{Conclusions}

We have presented new radial velocities and metallicities derived from high resolution spectra for five old distant anti-center open clusters previously unstudied. These results have been combined with similar quality data taken from the literature for eight additional old open clusters. We discuss this material in the context of the chemical and kinematic properties of the Galactic disk, with the principal aim to understand whether these clusters are genuine disk members, or that some of the clusters trace an extragalactic population which merged into the disk a few Gyr ago, the MRi.

Our results can be summarized as follows.

- the spatial location of these clusters is compatible with a warped/flared Galactic disk and poorly matches MRi model predictions;

- metallicities are compatible both with the Galactic disk and with the $\mathrm{M}$ giants which trace $\mathrm{MRi}$, whilst the abundance ratios of $\alpha$ elements are more compatible with the Galactic disk population;

- the AMR is in better agreement with the Galactic disk than with $\mathrm{MRi}$, although this latter is at present poorly constrained;

- we find that the cluster kinematics are difficult to interpret, in the sense that clusters seem to obey the Galactic differential rotation, but with increasing deviations at increasing distances. However, it is difficult with present data to disentangle whether these deviations are indicating an extragalactic origin, or are simply a consequence of the complicated dynamics in a warped/flared disk. In fact, in this case the expected increase of the velocity vertical component would naturally produce deviations from purely circular orbits;

- Models of MRi do not reproduce satisfactorily and simultaneously the position, radial velocities and Galactic standard of rest velocities $v_{\mathrm{GSR}}$ of the outer disk old open clusters here considered.

- the radial abundance gradients, especially those for the $\alpha-$ elements, are in good agreement with prediction based on simple Galactic disk models (Cescutti et al. 2006). We confirm that in the outer disk the radial iron abundance gradient is flatter than in the solar vicinity.

This work emphasizes the difficulty to study the periphery of the Galactic disk. A better description of the properties of the warped/flared disk, and of the Monoceros Ring models are mandatory to improve the comparison with the observations that we are accumulating. At the same time, the proper motion components of these clusters might greatly help to understand their kinematic properties, especially near the Galactic anti-center. The derivations of their orbits and eccentricities would constitute an important step ahead in our knowledge of the outer Galactic disk.

Acknowledgements. G.C. acknowledges fruitful discussions with Tom Richtler and thanks Gabriele Cescutti for calculating the slope of the radial abundance gradient for several elements. D.G. gratefully acknowledges support from the
Chilean Centro de Astrofísica FONDAP No. 15010003. G.C. and D.G. acknowledge support from the Padova and Concepción Universities exchange program. P.M.F. is supported by an NSF Astronomy and Astrophysics Postdoctoral Fellowship under award AST-0602221. Finally, the comments of the anonymous referee have been greatly appreciated.

\section{References}

Alonso, A., Arribas, S., \& Martńez-Roger, C. 1999, A\&AS, 140, 261 Bensby, T., Feltzing, S., \& Lundstrom, I. 2004, A\&A, 421, 969 Brewer, M.-M., \& Carney, B. W. 2006, AJ, 131, 431

Carney, B. W., Yong, D., Teixera de Almeida, M. L., \& Seitzer, P. 2005, AJ, 130, 1111

Carraro, G., \& Chiosi, C. 1994, A\&A, 288, 751

Carraro, G., Ng, Y. K., \& Portinari, L. 1998, MNRAS, 196, 1045

Carraro, G., Girardi, L., \& Chiosi, C. 1999, MNRAS, 309, 430

Carraro, G., Bresolin, F., Villanova, S., et al. 2004, AJ, 128, 1676

Carraro, G., Geisler, D., Moitinho, A., Baume, G., \& Vázquez, R. A. 2005a, A\&A, 442, 917

Carraro, G., Geisler, D., Baume, G., Vázquez, R. A., \& Moitinho, A. 2005b, MNRAS, 360, 655

Carraro, G., Subramaniam, A., \& Janes, K. A. 2006, MNRAS, 371, 1301

Casetti-Dinescu, D. I., Girard, T. M., Herrera, D., et al. 2007, AJ, in press [arXiv:0705.3438]

Cescutti, G., Matteucci, F., Francois, P., \& Chiappini, C. 2007, A\&A, 462, 943

Crane, J. D., Majewski, S. R., Rocha-Pinto, H. J., et al. 2003, ApJ, 594, L122

Friel, E. D. 1995, ARA\&A, 33, 381

Friel, E. D., Janes, K. A., Tavarez, M., et al. 2002, AJ, 124, 2693

Friel, E. D., Jacobson, H. R., Barrett, E., et al. 2003, AJ, 126, 2372

Finchaboy, P. M. 2006, in Globular Clusters- Guides to Galaxies, ed. T. Richtler, \& S. Larsen (ESO/Springer Conference Proceedings)

Frinchaboy, P. M., \& Phelps, R. L. 2002, AJ, 123, 2552

Frinchaboy, P. M., Majewski, S. R., Crane, J. D., et al. 2004, ApJ, 602, L21

Frinchaboy, P. M., Munoz, R. R., Phelps, R. L., et al. 2006, AJ, 131, 922

Frinchaboy, P. M., Marino, A. F., Villanova, S., et al. 2007, MNRAS, submitted Girardi, L., Bressan, A., Bertelli, G., \& Chiosi, C. 2000, A\&AS, 114, 371

Gratton, R., Carretta, E., Claudi, R., Lucatello, S., \& Barbieri, M. 2003, A\&A, 404, 187

Hill, V., \& Pasquini, L. 1999, A\&A, 348, L21

Houdashelt, M. L., Bell, R. A., \& Sweigart, A. V. 2000, AJ, 119, 1448

Ibata, R. A., Irwin, M. J., Lewis, G. F., Ferguson, A. M. N., \& Tanvir, N. 2003, MNRAS, 340, L21

Kurucz, R. L., Furenlid, I., Brault, J., \& Testerman, L. Solar Flux Atlas from 296 to $1300 \mathrm{~nm}$, National Solar Observatory Atlas No. 1, June 1984

Kurucz, R. L. 1992, in IAU Symp., 149, The Stellar Populations of Galaxies, ed. B. Barbuy, \& A. Renzini (Dordrecht: Reidel), 225

Layden, A. C., \& Sarajedini, A. 2000, AJ, 119, 1760

Majewski, S. R., Skrutskie, M. F., Weinberg, M. D., \& Ostheimer, J. C. 2003, ApJ, 599, 1082

Moitinho, A. 2001, A\&A, 370, 436

Moitinho, A., Vázquez, R. A., Carraro, G., et al. 2006, MNRAS, 368, L77

Momany, Y., Zaggia, S., Gilmore, G., et al. 2006, A\&A, 451, 515

Moustakas, J., \& Kennicut, R. C. 2006, ApJ, 651, 155

Newberg, H. J., Yanny, B., Rockosi, C., et al. 2002, ApJ, 569, 245

Norris, J. E. 1991, Ap\&SS, 265, 213

Olling, R. P., \& Dehnen, W. 2003, ApJ, 599, 275

Olling, R. P., \& Merrifield, M. R. 2000, MNRAS, 311, 361

Peñarrubia, J., Martínez-Delgado, D., Rix, H. W., et al. 2005, ApJ, 626, 128

Phelps, R. L., Janes, K. A., \& Montgomery, K. A. 1994, AJ, 107, 1079

Ramírez, I., Allende Prieto, C., \& Lambert, D. L. 2007, A\&A, 465, 271

Reddy, B. E., Tomkin, J., Lamber, D. L., Allende, \& Prieto, C. 2003, MNRAS, 304,340

Rocha-Pinto, H. J., Majewski, S. R., Skrutskie, M. F., \& Crane, J. D. 2003, ApJ, 594, L115

Sbordone, L., Bonifacio, P., Buonanno, R., Marconi, G., \& Zaggia, S. 2007, A\&A, 465, 815

Scott, J. E., Friel, E. D., \& Janes, K. A. 1995, AJ, 109, 1706

Sneden, C. A. 1973, ApJ, 184, 839

Twarog, B. A., Ashman, K. M., \& Anthony-Twarog, B. J. 1997, AJ, 114, 2556

Villanova, S., Carraro, G., Bresolin, F., \& Patat, F. 2005, AJ, 130, 652

Wilhelm, R., Beers, T. C., Allende Prieto, C., Newberg, H. J., \& Yanny, B. 2005, ASPC, 336, 371

Worthey, G., \& Lee, H.-C. 2006, ApJS, in press [arXiv:astro-ph/0604590]

Yanny, B., et al. 2003, ApJ, 588, 824

Yong, D., Carney, B. W., \& Teixera de Almeida 2005, AJ, 130, 597

Yong, D., Carney, B. W., Teixera de Almeida, M. L., \& Pohl, B. L. 2006, AJ, 130, 2256 
G. Carraro et al.: Old open clusters in the outer Galactic disk, Online Material $p 1$

\section{Online Material}


G. Carraro et al.: Old open clusters in the outer Galactic disk, Online Material p 2

Table 3.

\begin{tabular}{|c|c|c|c|c|c|c|c|c|}
\hline $\bar{\lambda}$ & $\overline{E l}$ & $\overline{\mathrm{EP}}$ & $\log (\mathrm{gf})$ & $\overline{\mathrm{Be} 25-12}$ & $\overline{\text { Be73-18 }}$ & $\overline{\mathrm{Be} 75-22}$ & $\begin{array}{c}\text { Rup4-3 } \\
\end{array}$ & $\overline{\text { Rup7-2 }}$ \\
\hline 4801.031 & 24.0 & 3.120 & -0.130 & 75.1 & 78.7 & 54.0 & 85.3 & 65.3 \\
\hline 4810.537 & 30.0 & 4.080 & -0.170 & 60.6 & 75.6 & 80.6 & 99.6 & 74.5 \\
\hline 4812.352 & 24.1 & 3.860 & -1.800 & - & - & 53.0 & 71.3 & 51.2 \\
\hline 4813.479 & 27.0 & 3.200 & 0.180 & - & 68.9 & 93.7 & 70.5 & 48.0 \\
\hline 4820.414 & 22.0 & 1.500 & -0.440 & 98.7 & 106.8 & 71.0 & 106.6 & 67.9 \\
\hline 4827.457 & 23.0 & 0.040 & -1.480 & - & - & - & 74.2 & - \\
\hline 4831.651 & 23.0 & 0.020 & -1.380 & 90.0 & 74.1 & - & 57.0 & - \\
\hline 4832.431 & 23.0 & 0.000 & -1.500 & 104.5 & - & - & - & - \\
\hline 4836.857 & 24.0 & 3.100 & -1.140 & 71.6 & - & - & - & - \\
\hline 4848.252 & 24.1 & 3.860 & -1.080 & - & - & 80.4 & 102.0 & 84.1 \\
\hline 4854.873 & 39.1 & 0.990 & -0.380 & 74.4 & 87.9 & 78.2 & 100.5 & 88.2 \\
\hline 4864.738 & 23.0 & 0.020 & -0.960 & 97.0 & 99.2 & 121.5 & 83.0 & 52.7 \\
\hline 4865.618 & 22.1 & 1.120 & -2.750 & 94.6 & 86.9 & - & 95.4 & 76.6 \\
\hline 4867.874 & 27.0 & 3.100 & 0.410 & 107.7 & 117.7 & 63.4 & 98.3 & - \\
\hline 4874.014 & 22.1 & 3.090 & -0.940 & - & 46.1 & - & 56.1 & 52.2 \\
\hline 4875.492 & 23.0 & 0.040 & -0.810 & 95.0 & 102.2 & 84.7 & 90.6 & 64.7 \\
\hline 4883.690 & 39.1 & 1.080 & 0.070 & 85.5 & 104.2 & 74.0 & 102.2 & 109.9 \\
\hline 4900.124 & 39.1 & 1.030 & -0.090 & - & - & - & - & 175.1 \\
\hline 4904.420 & 28.0 & 3.540 & -0.190 & - & 115.4 & 81.5 & 110.9 & 91.2 \\
\hline 4911.199 & 22.1 & 3.120 & -0.490 & - & 67.7 & 63.3 & 87.9 & 73.6 \\
\hline 4913.978 & 28.0 & 3.740 & -0.630 & 67.0 & 76.3 & - & 73.0 & 61.2 \\
\hline 4935.834 & 28.0 & 3.940 & -0.380 & 79.6 & 77.8 & 45.3 & 75.4 & 59.3 \\
\hline 4936.341 & 24.0 & 3.110 & -0.220 & 60.2 & 71.1 & 51.7 & 66.5 & 46.3 \\
\hline 4953.212 & 28.0 & 3.740 & -0.640 & 110.8 & 100.7 & 89.4 & 82.5 & 58.7 \\
\hline 4954.809 & 24.0 & 3.120 & -0.140 & - & - & - & - & 73.6 \\
\hline 4964.933 & 24.0 & 0.940 & -2.530 & 111.5 & 93.6 & 78.3 & - & - \\
\hline 4981.740 & 22.0 & 0.850 & 0.400 & 163.7 & 152.6 & 159.6 & 160.7 & 86.2 \\
\hline 4997.100 & 22.0 & 0.000 & -2.120 & 95.8 & 98.1 & 65.8 & - & - \\
\hline 4999.510 & 22.0 & 0.830 & 0.250 & 167.4 & 162.2 & 135.9 & 169.0 & 140.2 \\
\hline 5003.747 & 28.0 & 1.680 & -3.130 & 88.3 & 59.6 & 44.4 & 61.4 & - \\
\hline 5004.894 & 25.0 & 2.920 & -1.650 & - & 36.9 & - & - & - \\
\hline 5005.171 & 22.1 & 1.570 & -2.680 & - & 56.4 & 37.6 & 46.7 & 47.1 \\
\hline 5009.655 & 22.0 & 0.020 & -2.260 & 117.1 & 83.8 & 68.8 & 77.7 & - \\
\hline 5010.943 & 28.0 & 3.630 & -0.900 & - & 64.2 & 52.5 & 69.4 & 52.3 \\
\hline 5016.168 & 22.0 & 0.850 & -0.570 & 122.5 & 101.7 & 90.3 & 100.9 & 76.2 \\
\hline 5022.874 & 22.0 & 0.830 & -0.430 & 107.5 & 119.6 & 106.7 & 114.8 & 94.0 \\
\hline 5039.964 & 22.0 & 0.020 & -1.230 & 144.1 & 135.8 & 107.3 & 123.8 & 94.4 \\
\hline 5048.853 & 28.0 & 3.850 & -0.390 & 124.3 & 107.6 & - & 102.0 & 70.2 \\
\hline 5062.104 & 22.0 & 2.160 & -0.440 & - & 45.2 & - & 36.5 & - \\
\hline 5087.426 & 39.1 & 1.080 & -0.170 & 65.8 & 74.0 & 59.8 & 92.9 & 82.5 \\
\hline 5094.418 & 28.0 & 3.830 & -1.120 & - & 41.5 & - & 42.5 & 30.5 \\
\hline 5105.545 & 29.0 & 1.380 & -1.020 & 159.4 & 136.8 & 127.2 & 139.6 & 105.1 \\
\hline 5112.279 & 40.1 & 1.660 & -0.590 & - & - & - & 47.2 & - \\
\hline 5113.447 & 22.0 & 1.440 & -0.780 & - & 71.6 & 57.3 & 61.3 & - \\
\hline 5145.468 & 22.0 & 1.460 & -0.570 & 96.2 & 68.5 & 71.8 & 69.8 & 44.7 \\
\hline 5147.482 & 22.0 & 0.000 & -2.010 & 106.8 & 85.4 & - & 87.8 & 61.8 \\
\hline 5152.190 & 22.0 & 0.020 & -2.020 & 94.2 & 92.8 & 88.5 & - & - \\
\hline 5157.984 & 28.0 & 3.510 & -1.720 & - & - & - & 27.2 & - \\
\hline 5173.750 & 22.0 & 0.000 & -1.370 & 95.5 & 90.8 & 115.9 & 91.9 & 102.9 \\
\hline 5185.908 & 22.1 & 1.890 & -1.500 & 93.9 & 102.9 & 86.0 & 109.2 & 101.0 \\
\hline 5197.170 & 28.0 & 3.900 & -1.140 & - & 50.2 & - & 58.0 & - \\
\hline 5200.185 & 24.0 & 3.380 & -0.530 & - & - & 51.9 & - & - \\
\hline 5200.415 & 39.1 & 0.990 & -0.570 & 97.3 & 91.1 & 71.0 & 99.9 & - \\
\hline 5210.392 & 22.0 & 0.050 & -0.880 & 158.6 & 142.8 & 116.2 & 137.1 & 104.8 \\
\hline 5212.691 & 27.0 & 3.510 & -0.110 & - & - & - & 33.8 & - \\
\hline 5214.616 & 24.0 & 3.320 & -0.660 & - & - & - & 48.5 & - \\
\hline 5218.209 & 29.0 & 3.800 & 0.300 & 85.5 & 63.9 & 61.7 & 78.3 & - \\
\hline 5219.706 & 22.0 & 0.020 & -2.260 & 96.2 & 83.0 & 57.5 & 75.7 & 47.3 \\
\hline 5237.325 & 24.1 & 4.070 & -1.170 & - & 75.0 & 67.4 & 92.2 & 72.2 \\
\hline 5238.969 & 24.0 & 2.710 & -1.300 & - & - & - & 35.9 & - \\
\hline
\end{tabular}


G. Carraro et al.: Old open clusters in the outer Galactic disk, Online Material $p 3$

Table 3. continued.

\begin{tabular}{|c|c|c|c|c|c|c|c|c|}
\hline $\bar{\lambda}$ & $\overline{E l}$ & $\overline{E P}$ & $\log (\mathrm{gf})$ & $\overline{\mathrm{Be} 25-12}$ & $\overline{\text { Be73-18 }}$ & $\overline{B e 75-22}$ & Rup4-3 & Rup7-2 \\
\hline 5239.823 & 21.1 & 1.450 & -0.700 & 92.4 & 112.2 & 65.9 & 107.0 & 93.1 \\
\hline 5247.574 & 24.0 & 0.960 & -1.630 & 133.7 & 118.6 & 80.7 & 114.0 & - \\
\hline 5260.390 & 20.0 & 2.520 & -1.720 & 68.7 & 49.5 & - & 52.1 & - \\
\hline 5261.708 & 20.0 & 2.520 & -0.580 & 113.8 & 123.4 & 112.3 & 126.5 & 104.4 \\
\hline 5272.003 & 24.0 & 3.450 & -0.420 & - & - & - & 40.3 & - \\
\hline 5274.236 & 58.1 & 1.040 & 0.260 & - & 33.3 & - & 44.0 & - \\
\hline 5282.443 & 22.0 & 1.050 & -1.300 & 57.5 & 48.4 & 51.4 & 44.5 & 24.7 \\
\hline 5295.784 & 22.0 & 1.050 & -1.630 & 70.5 & 45.1 & - & 40.8 & - \\
\hline 5296.702 & 24.0 & 0.980 & -1.240 & 154.5 & 138.8 & 113.6 & 140.1 & 109.7 \\
\hline 5299.984 & 22.0 & 1.050 & -1.360 & - & 41.6 & - & 33.2 & - \\
\hline 5300.751 & 24.0 & 0.980 & -2.130 & 109.3 & 96.5 & 75.0 & 97.6 & - \\
\hline 5304.185 & 24.0 & 3.460 & -0.690 & 38.9 & - & _- & - & - \\
\hline 5305.866 & 24.1 & 3.830 & -2.080 & - & 36.7 & - & 50.2 & 46.4 \\
\hline 5308.429 & 24.1 & 4.070 & -1.850 & - & - & - & 53.7 & 48.2 \\
\hline 5310.697 & 24.1 & 4.070 & -2.280 & - & - & - & 29.1 & - \\
\hline 5312.863 & 24.0 & 3.450 & -0.560 & - & - & - & 36.0 & 24.2 \\
\hline 5313.585 & 24.1 & 4.070 & -1.640 & 44.9 & - & - & 62.1 & 61.4 \\
\hline 5318.361 & 21.1 & 1.360 & -1.750 & - & - & - & 46.5 & 43.0 \\
\hline 5319.820 & 60.1 & 0.550 & -0.560 & - & - & 51.3 & - & 79.1 \\
\hline 5336.790 & 22.1 & 1.580 & -1.590 & 112.3 & 100.1 & 86.3 & 132.7 & 121.6 \\
\hline 5342.708 & 27.0 & 4.020 & 0.520 & - & 51.7 & 41.0 & 48.4 & 31.7 \\
\hline 5345.807 & 24.0 & 1.000 & -0.970 & 199.0 & 166.8 & 152.2 & 168.3 & 137.2 \\
\hline 5348.326 & 24.0 & 1.000 & -1.290 & 170.6 & 141.5 & 123.0 & 134.4 & 114.6 \\
\hline 5349.469 & 20.0 & 2.710 & -0.460 & 105.1 & 112.6 & 87.6 & 120.4 & - \\
\hline 5352.049 & 27.0 & 3.560 & 0.060 & - & 50.2 & - & 47.9 & - \\
\hline 5402.783 & 39.1 & 1.840 & -0.640 & - & - & - & 44.8 & 49.3 \\
\hline 5409.799 & 24.0 & 1.030 & -0.710 & 178.7 & 184.2 & 164.2 & 184.9 & 142.0 \\
\hline 5418.780 & 22.1 & 1.580 & -2.110 & - & 81.0 & 61.9 & 93.3 & 84.2 \\
\hline 5432.548 & 25.0 & 0.000 & -3.800 & 153.8 & 127.0 & 117.4 & 111.7 & 85.9 \\
\hline 5490.159 & 22.0 & 1.460 & -0.930 & 71.5 & 64.0 & - & 57.0 & - \\
\hline 5494.474 & 26.0 & 4.070 & -1.960 & - & 44.6 & - & 46.4 & - \\
\hline 5502.092 & 24.1 & 4.170 & -1.960 & - & - & - & 36.2 & 40.6 \\
\hline 5503.904 & 22.0 & 2.580 & -0.190 & 48.6 & - & - & - & - \\
\hline 5522.454 & 26.0 & 4.210 & -1.470 & 64.1 & 57.2 & - & 60.3 & 47.8 \\
\hline 5525.135 & 26.1 & 3.270 & -4.040 & - & - & - & 32.2 & - \\
\hline 5526.821 & 21.1 & 1.770 & 0.190 & 101.3 & 116.6 & 87.9 & 123.4 & 110.7 \\
\hline 5534.848 & 26.1 & 3.240 & -2.750 & - & 67.0 & 42.5 & 102.4 & 101.4 \\
\hline 5539.291 & 26.0 & 3.640 & -2.590 & - & 35.7 & - & 35.6 & - \\
\hline 5547.000 & 26.0 & 4.220 & -1.800 & 57.1 & 44.0 & - & 54.2 & 31.6 \\
\hline 5560.220 & 26.0 & 4.430 & -1.100 & 66.5 & 66.8 & 51.8 & 70.9 & 49.9 \\
\hline 5568.862 & 26.0 & 3.630 & -2.910 & - & - & - & 25.9 & - \\
\hline 5578.729 & 28.0 & 1.680 & -2.570 & 114.5 & 89.8 & 106.0 & 102.1 & 72.9 \\
\hline 5587.581 & 26.0 & 4.140 & -1.700 & - & 51.5 & 31.0 & - & - \\
\hline 5587.868 & 28.0 & 1.930 & -2.390 & 95.8 & 81.4 & 58.3 & 97.1 & - \\
\hline 5589.366 & 28.0 & 3.900 & -1.150 & - & 42.4 & 25.6 & - & - \\
\hline 5590.126 & 20.0 & 2.510 & -0.770 & 101.2 & 111.4 & 92.4 & 118.8 & 95.2 \\
\hline 5593.746 & 28.0 & 3.900 & -0.780 & - & 74.7 & - & 54.4 & 44.7 \\
\hline 5608.976 & 26.0 & 4.210 & -2.310 & - & - & - & 21.7 & 16.8 \\
\hline 5611.357 & 26.0 & 3.630 & -2.930 & 34.4 & - & - & - & - \\
\hline 5618.642 & 26.0 & 4.210 & -1.340 & 67.1 & 70.2 & 62.1 & 65.6 & 50.8 \\
\hline 5619.609 & 26.0 & 4.390 & -1.490 & 54.7 & 59.7 & - & 45.4 & 33.5 \\
\hline 5627.642 & 23.0 & 1.080 & -0.370 & 106.5 & 74.5 & 55.6 & 69.8 & 57.8 \\
\hline 5635.831 & 26.0 & 4.260 & -1.590 & - & 43.0 & - & 49.5 & 35.0 \\
\hline 5636.705 & 26.0 & 3.640 & -2.530 & - & 29.6 & 26.8 & 35.5 & - \\
\hline 5640.989 & 21.1 & 1.500 & -0.860 & 86.0 & 87.3 & 62.8 & 97.7 & 81.0 \\
\hline 5643.087 & 28.0 & 4.160 & -1.250 & - & - & - & 28.2 & - \\
\hline 5645.618 & 14.0 & 4.930 & -2.140 & - & 56.1 & - & - & - \\
\hline 5647.241 & 27.0 & 2.280 & -1.560 & 68.6 & 33.1 & - & 37.6 & - \\
\hline 5649.996 & 26.0 & 5.100 & -0.800 & 52.5 & 46.5 & - & 57.8 & - \\
\hline 5651.477 & 26.0 & 4.470 & -1.790 & - & - & - & 36.5 & 20.9 \\
\hline
\end{tabular}


Table 3. continued.

\begin{tabular}{|c|c|c|c|c|c|c|c|c|}
\hline$\overline{\bar{\lambda}}$ & $\overline{\mathrm{El}}$ & $\overline{\overline{\mathrm{EP}}}$ & $\overline{\log (\mathrm{gf})}$ & $\overline{\mathrm{Be} 25-12}$ & $\overline{\mathrm{B} \text { Be73-18 }}$ & 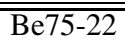 & $\overline{\text { Rup4-3 }}$ & Rup7-2 \\
\hline 5652.327 & 26.0 & 4.260 & -1.770 & - & 46.3 & - & 48.3 & 30.6 \\
\hline 5657.880 & 21.1 & 1.510 & -0.290 & 125.5 & 105.5 & 98.3 & 115.1 & 107.3 \\
\hline 5661.354 & 26.0 & 4.280 & -1.830 & 45.5 & 54.6 & - & 40.6 & 28.1 \\
\hline 5662.159 & 22.0 & 2.320 & -0.110 & - & 49.2 & - & - & - \\
\hline 5665.563 & 14.0 & 4.920 & -2.040 & 44.5 & - & 46.2 & 59.2 & 51.5 \\
\hline 5667.153 & 21.1 & 1.500 & -1.110 & 62.7 & 71.2 & 57.8 & 78.3 & - \\
\hline 5669.040 & 21.1 & 1.500 & -1.000 & 72.4 & 78.3 & - & 92.5 & 92.2 \\
\hline 5670.858 & 23.0 & 1.080 & -0.420 & 92.3 & 69.7 & - & 48.3 & 28.7 \\
\hline 5671.826 & 21.0 & 1.450 & 0.560 & - & - & 37.7 & 35.5 & - \\
\hline 5680.244 & 26.0 & 4.190 & -2.290 & - & - & - & 26.1 & - \\
\hline 5682.647 & 11.0 & 2.100 & -0.870 & - & 129.3 & 110.2 & 131.8 & 109.4 \\
\hline 5684.198 & 21.1 & 1.510 & -0.920 & 79.0 & - & 95.7 & - & - \\
\hline 5688.217 & 11.0 & 2.100 & -0.570 & 129.6 & 135.3 & 128.2 & 146.3 & - \\
\hline 5690.433 & 14.0 & 4.930 & -1.870 & 61.8 & 61.5 & - & 63.4 & 55.0 \\
\hline 5698.530 & 23.0 & 1.060 & -0.110 & 128.1 & - & 82.9 & - & - \\
\hline 5701.108 & 14.0 & 4.930 & -2.050 & - & 45.7 & - & 44.8 & - \\
\hline 5701.557 & 26.0 & 2.560 & -2.160 & 122.0 & 123.6 & 103.1 & 112.4 & 88.6 \\
\hline 5702.328 & 24.0 & 3.450 & -0.480 & 53.8 & 53.7 & - & 40.9 & 30.9 \\
\hline 5703.587 & 23.0 & 1.050 & -0.210 & 117.8 & 76.5 & 61.5 & 60.7 & 40.6 \\
\hline 5708.405 & 14.0 & 4.950 & -1.470 & 80.9 & - & - & - & - \\
\hline 5711.095 & 12.0 & 4.340 & -1.710 & 124.9 & 116.4 & 135.8 & 117.9 & 87.8 \\
\hline 5717.841 & 26.0 & 4.280 & -0.980 & 91.4 & 77.1 & 65.0 & 89.4 & - \\
\hline 5727.057 & 23.0 & 1.080 & -0.010 & 102.6 & 85.7 & 81.6 & 81.7 & - \\
\hline 5731.772 & 26.0 & 4.260 & -1.000 & - & 68.8 & 78.3 & 79.4 & 64.4 \\
\hline 5741.856 & 26.0 & 4.260 & -1.690 & - & 51.5 & - & 42.1 & 39.2 \\
\hline 5748.361 & 28.0 & 1.680 & -3.250 & - & - & - & - & 52.1 \\
\hline 5752.042 & 26.0 & 4.550 & -0.920 & 33.1 & - & 37.5 & - & 49.4 \\
\hline 5760.359 & 26.0 & 3.640 & -2.460 & - & - & - & - & 30.4 \\
\hline 5772.149 & 14.0 & 5.080 & -1.750 & - & - & 27.3 & - & 52.3 \\
\hline 5847.006 & 28.0 & 1.680 & -3.440 & 81.7 & 65.8 & - & 50.7 & 40.5 \\
\hline 5852.228 & 26.0 & 4.550 & -1.160 & - & 66.0 & - & 63.0 & 50.2 \\
\hline 5853.150 & 26.0 & 1.480 & -5.090 & 49.5 & 39.6 & - & - & - \\
\hline 5853.688 & 56.1 & 0.600 & -1.000 & 112.6 & 112.4 & 120.4 & 143.1 & 150.5 \\
\hline 5855.086 & 26.0 & 4.610 & -1.560 & 52.0 & - & 35.8 & 40.6 & 24.0 \\
\hline 5856.096 & 26.0 & 4.290 & -1.570 & - & 52.1 & 57.7 & 58.4 & 36.0 \\
\hline 5858.785 & 26.0 & 4.220 & -2.190 & - & - & - & 20.3 & - \\
\hline 5859.596 & 26.0 & 4.550 & -0.630 & 86.6 & 75.6 & 63.5 & 91.2 & 71.9 \\
\hline 5861.110 & 26.0 & 4.280 & -2.350 & - & - & - & 19.7 & 13.1 \\
\hline 5862.368 & 26.0 & 4.550 & -0.420 & 107.0 & 101.3 & 86.5 & 101.0 & 85.9 \\
\hline 5866.461 & 22.0 & 1.070 & -0.840 & 115.9 & 105.1 & 99.6 & 88.9 & 65.7 \\
\hline 5867.572 & 20.0 & 2.930 & -1.490 & - & 34.6 & - & 35.6 & 25.5 \\
\hline 5881.279 & 26.0 & 4.610 & -1.760 & 33.6 & - & - & 25.1 & - \\
\hline 5902.476 & 26.0 & 4.590 & -1.860 & - & - & - & 20.0 & - \\
\hline 5905.680 & 26.0 & 4.650 & -0.780 & - & 59.7 & 81.4 & 76.7 & 62.8 \\
\hline 5922.123 & 22.0 & 1.050 & -1.470 & 100.7 & 71.6 & 73.3 & 52.9 & - \\
\hline 5927.797 & 26.0 & 4.650 & -1.070 & 91.4 & 52.0 & 60.8 & 61.6 & 48.6 \\
\hline 5929.682 & 26.0 & 4.550 & -1.160 & - & 48.7 & - & 54.1 & 46.3 \\
\hline 5930.191 & 26.0 & 4.650 & -0.340 & 126.0 & 93.0 & 84.3 & 102.2 & 84.9 \\
\hline 5934.665 & 26.0 & 3.930 & -1.080 & 100.0 & 91.5 & 78.1 & 101.9 & 86.7 \\
\hline 5947.531 & 26.0 & 4.610 & -2.040 & - & - & - & 16.9 & - \\
\hline 5948.548 & 14.0 & 5.080 & -1.230 & - & 92.4 & 67.5 & 110.9 & 88.0 \\
\hline 5956.706 & 26.0 & 0.860 & -4.560 & 100.0 & 113.0 & 62.9 & 98.9 & 87.4 \\
\hline 5978.549 & 22.0 & 1.870 & -0.580 & - & 50.1 & - & 51.0 & 33.5 \\
\hline 5984.826 & 26.0 & 4.730 & -0.290 & - & 79.6 & 89.8 & 90.2 & 82.8 \\
\hline 5991.378 & 26.1 & 3.150 & -3.550 & 45.7 & 42.4 & 39.8 & 60.8 & 60.3 \\
\hline 5996.740 & 28.0 & 4.230 & -1.060 & - & 36.1 & 39.2 & 29.5 & 20.4 \\
\hline 6003.022 & 26.0 & 3.880 & -1.020 & 93.0 & 99.3 & 111.9 & 105.3 & 89.5 \\
\hline 6007.968 & 26.0 & 4.650 & -0.760 & 73.5 & 70.8 & - & 76.0 & 61.6 \\
\hline 6008.566 & 26.0 & 3.880 & -0.920 & 126.6 & 104.2 & 92.1 & 98.4 & 92.2 \\
\hline 6013.497 & 25.0 & 3.070 & -0.250 & 120.2 & 117.7 & 94.9 & 112.4 & 82.8 \\
\hline 6016.647 & 25.0 & 3.070 & -0.090 & - & 129.2 & 105.2 & 113.6 & 107.6 \\
\hline 6021.803 & 25.0 & 3.080 & 0.030 & 151.4 & 131.1 & 120.6 & 119.2 & 98.6 \\
\hline
\end{tabular}


G. Carraro et al.: Old open clusters in the outer Galactic disk, Online Material p 5

Table 3. continued.

\begin{tabular}{|c|c|c|c|c|c|c|c|c|}
\hline $\bar{\lambda}$ & $\overline{\overline{\text { El }}}$ & $\overline{\overline{\mathrm{EP}}}$ & $\overline{\log (\mathrm{gf})}$ & $\overline{\mathrm{Be} 25-12}$ & 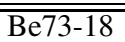 & $\overline{\mathrm{B} \text { Be75-22 }}$ & "Rup4-3 & $\overline{\text { Rup7-2 }}$ \\
\hline 6027.059 & 26.0 & 4.070 & -1.200 & 104.2 & 83.2 & 71.5 & 100.3 & 76.7 \\
\hline 6053.693 & 28.0 & 4.230 & -1.070 & - & - & - & 27.5 & - \\
\hline 6056.013 & 26.0 & 4.730 & -0.460 & 82.9 & 81.1 & 70.0 & 87.4 & 68.1 \\
\hline 6065.494 & 26.0 & 2.610 & -1.590 & 148.3 & 157.2 & 139.1 & 163.6 & - \\
\hline 6078.499 & 26.0 & 4.790 & -0.380 & - & 77.7 & 67.9 & 97.6 & 80.7 \\
\hline 6079.016 & 26.0 & 4.650 & -0.970 & - & - & 80.7 & 65.0 & 41.3 \\
\hline 6081.448 & 23.0 & 1.050 & -0.580 & - & - & 74.2 & - & 32.2 \\
\hline 6082.718 & 26.0 & 2.220 & -3.530 & 82.1 & 78.2 & 61.9 & 73.7 & 59.5 \\
\hline 6084.105 & 26.1 & 3.200 & -3.800 & - & - & - & 50.0 & 61.8 \\
\hline 6086.288 & 28.0 & 4.260 & -0.470 & - & 50.4 & - & 53.9 & 47.5 \\
\hline 6089.574 & 26.0 & 5.020 & -0.870 & 58.9 & 53.8 & - & 66.8 & 47.2 \\
\hline 6090.216 & 23.0 & 1.080 & -0.060 & 85.4 & 85.1 & 57.8 & 68.9 & 59.9 \\
\hline 6091.177 & 22.0 & 2.270 & -0.420 & - & 36.5 & - & 38.5 & 19.9 \\
\hline 6093.649 & 26.0 & 4.610 & -1.320 & - & 42.4 & - & 48.7 & 36.7 \\
\hline 6094.377 & 26.0 & 4.650 & -1.560 & - & 32.2 & - & 36.1 & 18.9 \\
\hline 6096.671 & 26.0 & 3.980 & -1.760 & 58.0 & 63.5 & 72.4 & 61.2 & 44.6 \\
\hline 6098.250 & 26.0 & 4.560 & -1.810 & - & - & 27.4 & 38.9 & - \\
\hline 6108.125 & 28.0 & 1.680 & -2.470 & 98.8 & 102.5 & 89.5 & 107.1 & 85.1 \\
\hline 6111.078 & 28.0 & 4.090 & -0.830 & - & 51.9 & 45.6 & 50.9 & 31.7 \\
\hline 6113.329 & 26.1 & 3.210 & -4.120 & - & - & - & 37.4 & - \\
\hline 6119.532 & 23.0 & 1.060 & -0.320 & 99.6 & 59.4 & - & 58.6 & 42.1 \\
\hline 6120.258 & 26.0 & 0.910 & -5.860 & 57.2 & 28.0 & - & 27.5 & - \\
\hline 6125.026 & 14.0 & 5.610 & -1.570 & 49.2 & - & - & 39.4 & 34.9 \\
\hline 6126.224 & 22.0 & 1.070 & -1.420 & 94.7 & 73.2 & 57.9 & 57.9 & 44.5 \\
\hline 6128.984 & 28.0 & 1.680 & -3.390 & 85.4 & 74.5 & - & 62.7 & 49.0 \\
\hline 6130.141 & 28.0 & 4.260 & -0.980 & - & 43.5 & - & - & 25.5 \\
\hline 6137.002 & 26.0 & 2.200 & -2.910 & 106.7 & 104.9 & 99.7 & - & - \\
\hline 6145.020 & 14.0 & 5.610 & -1.440 & - & - & - & 45.8 & - \\
\hline 6149.249 & 26.1 & 3.890 & -2.720 & 53.4 & 39.2 & 37.7 & 61.4 & 69.8 \\
\hline 6151.623 & 26.0 & 2.180 & -3.260 & 114.9 & 83.8 & 83.1 & 95.5 & 71.7 \\
\hline 6154.230 & 11.0 & 2.100 & -1.570 & 67.8 & 51.7 & 60.6 & 62.9 & 46.1 \\
\hline 6157.733 & 26.0 & 4.070 & -1.260 & - & 96.1 & 77.2 & 91.9 & 80.7 \\
\hline 6160.753 & 11.0 & 2.100 & -1.260 & 69.7 & 80.7 & 55.7 & 80.7 & 67.3 \\
\hline 6161.295 & 20.0 & 2.520 & -1.270 & 76.0 & 79.8 & 75.4 & 87.0 & 80.1 \\
\hline 6165.363 & 26.0 & 4.140 & -1.480 & 66.4 & 64.3 & 61.3 & 59.8 & 54.2 \\
\hline 6166.440 & 20.0 & 2.520 & -1.140 & 97.5 & 87.7 & 83.3 & 91.2 & 72.2 \\
\hline 6169.044 & 20.0 & 2.520 & -0.800 & 136.6 & 93.2 & 103.3 & 112.0 & 100.7 \\
\hline 6169.564 & 20.0 & 2.520 & -0.480 & 151.9 & 121.0 & 123.3 & 123.1 & 105.7 \\
\hline 6173.341 & 26.0 & 2.220 & -2.840 & 107.5 & 104.1 & 79.5 & 107.7 & 92.9 \\
\hline 6176.816 & 28.0 & 4.090 & -0.240 & 69.0 & 79.6 & 70.5 & 76.7 & 62.7 \\
\hline 6177.253 & 28.0 & 1.830 & -3.600 & 52.2 & 41.5 & - & 33.7 & - \\
\hline 6186.717 & 28.0 & 4.100 & -0.900 & - & - & - & 43.7 & 34.0 \\
\hline 6187.995 & 26.0 & 3.940 & -1.600 & 76.3 & 66.8 & - & 72.1 & 55.2 \\
\hline 6199.186 & 23.0 & 0.290 & -1.280 & - & 73.6 & - & 41.8 & 33.5 \\
\hline 6200.321 & 26.0 & 2.610 & -2.390 & - & 111.0 & - & 114.3 & 97.2 \\
\hline 6204.610 & 28.0 & 4.090 & -1.150 & - & - & - & 40.9 & 29.3 \\
\hline 6213.437 & 26.0 & 2.220 & -2.540 & 130.8 & 131.2 & 94.5 & 122.4 & 109.0 \\
\hline 6216.358 & 23.0 & 0.280 & -0.810 & 109.3 & 82.7 & - & 66.9 & 46.4 \\
\hline 6219.287 & 26.0 & 2.200 & -2.390 & 113.5 & 125.6 & 102.3 & 135.1 & 120.6 \\
\hline 6223.990 & 28.0 & 4.100 & -0.970 & - & 37.9 & - & 40.3 & 28.9 \\
\hline 6226.740 & 26.0 & 3.880 & -2.080 & 43.3 & 44.1 & - & 40.2 & 33.1 \\
\hline 6230.098 & 28.0 & 4.100 & -1.200 & - & - & - & - & 26.0 \\
\hline 6232.648 & 26.0 & 3.650 & -1.060 & 113.4 & 79.0 & 100.0 & 113.0 & 96.0 \\
\hline 6240.653 & 26.0 & 2.220 & -3.230 & 99.3 & 88.5 & 77.2 & 90.1 & 81.9 \\
\hline 6243.114 & 23.0 & 0.300 & -0.980 & 152.2 & 97.0 & 85.4 & 82.1 & - \\
\hline 6245.620 & 21.1 & 1.510 & -1.050 & 61.6 & 75.9 & 55.6 & 76.9 & 82.6 \\
\hline 6246.327 & 26.0 & 3.600 & -0.730 & 154.9 & 123.2 & 113.4 & 134.7 & 119.2 \\
\hline 6247.562 & 26.1 & 3.870 & -2.320 & 57.9 & 60.8 & - & 65.7 & 92.7 \\
\hline 6251.825 & 23.0 & 0.290 & -1.340 & 112.2 & 71.4 & 43.0 & 51.7 & 29.3 \\
\hline 6252.565 & 26.0 & 2.400 & -1.740 & 159.7 & 175.2 & 122.9 & 166.4 & 142.5 \\
\hline 6258.110 & 22.0 & 1.440 & -0.360 & 98.9 & 94.1 & 76.0 & 86.2 & 65.4 \\
\hline 6261.106 & 22.0 & 1.430 & -0.480 & 145.5 & 105.0 & 82.2 & 94.7 & 74.0 \\
\hline
\end{tabular}


G. Carraro et al.: Old open clusters in the outer Galactic disk, Online Material p 6

Table 3. continued.

\begin{tabular}{|c|c|c|c|c|c|c|c|c|}
\hline$\lambda$ & El & $\overline{\mathrm{EP}}$ & $\log (\mathrm{gf})$ & Be25-12 & Be73-18 & Be75-22 & Rup4-3 & Rup7-2 \\
\hline 6265.141 & 26.0 & 2.180 & -2.510 & 161.3 & 133.5 & 99.9 & 133.5 & 124.5 \\
\hline 6270.231 & 26.0 & 2.860 & -2.550 & 108.8 & 91.8 & 79.0 & 92.3 & 77.1 \\
\hline 6279.740 & 21.1 & 1.500 & -1.160 & 55.3 & - & - & 78.6 & - \\
\hline 6280.622 & 26.0 & 0.860 & -4.340 & 110.6 & 114.0 & 93.0 & 135.7 & 101.7 \\
\hline 6297.799 & 26.0 & 2.220 & -2.700 & - & 127.3 & 113.7 & 125.3 & 130.5 \\
\hline 6300.311 & 8.0 & 0.000 & -9.750 & - & - & - & 23.2 & - \\
\hline 6301.508 & 26.0 & 3.650 & -0.570 & 126.6 & 120.3 & 124.6 & 146.4 & 129.0 \\
\hline 6311.504 & 26.0 & 2.830 & -3.160 & 104.1 & 58.0 & 44.4 & 62.0 & 47.7 \\
\hline 6315.814 & 26.0 & 4.070 & -1.670 & 74.1 & 64.2 & 47.9 & 66.9 & 50.1 \\
\hline 6318.708 & 12.0 & 5.110 & -1.970 & - & - & - & - & 38.1 \\
\hline 6322.694 & 26.0 & 2.590 & -2.380 & 132.8 & 109.6 & 97.3 & 109.0 & 96.0 \\
\hline 6327.604 & 28.0 & 1.680 & -3.080 & 68.5 & 91.4 & 61.2 & 76.2 & 71.1 \\
\hline 6330.096 & 24.0 & 0.940 & -2.870 & - & 71.9 & - & 72.0 & 54.8 \\
\hline 6330.852 & 26.0 & 4.730 & -1.220 & - & 54.3 & 71.9 & 49.7 & 43.9 \\
\hline 6335.337 & 26.0 & 2.200 & -2.280 & 145.9 & 137.8 & 117.1 & 135.9 & 126.6 \\
\hline 6369.463 & 26.1 & 2.890 & -4.210 & - & 25.6 & 37.6 & 44.7 & 37.4 \\
\hline 6378.256 & 28.0 & 4.150 & -0.820 & - & 43.7 & 49.0 & 46.1 & - \\
\hline 6380.750 & 26.0 & 4.190 & -1.340 & 88.6 & 71.7 & 54.4 & 81.2 & 63.9 \\
\hline 6383.715 & 26.1 & 5.550 & -2.090 & - & - & - & - & 15.4 \\
\hline 6384.668 & 28.0 & 4.150 & -1.000 & - & - & - & 38.0 & 28.8 \\
\hline 6392.538 & 26.0 & 2.280 & -3.970 & - & - & - & 45.4 & - \\
\hline 6393.612 & 26.0 & 2.430 & -1.630 & 176.7 & 192.0 & 131.1 & 171.0 & 151.5 \\
\hline 6411.658 & 26.0 & 3.650 & -0.700 & 146.6 & 125.8 & 111.1 & 139.9 & 125.7 \\
\hline 6416.928 & 26.1 & 3.890 & -2.700 & - & 47.0 & - & 61.9 & 64.1 \\
\hline 6432.683 & 26.1 & 2.890 & -3.580 & 41.0 & 53.3 & 57.0 & 73.6 & 76.6 \\
\hline 6436.411 & 26.0 & 4.190 & -2.400 & - & 30.6 & - & 25.9 & 15.1 \\
\hline 6439.083 & 20.0 & 2.520 & 0.190 & 159.8 & 172.7 & 158.2 & 189.5 & 162.8 \\
\hline 6455.605 & 20.0 & 2.520 & -1.290 & 119.2 & 75.0 & 45.3 & 89.7 & 66.7 \\
\hline 6456.391 & 26.1 & 3.900 & -2.100 & 72.7 & 67.0 & - & 88.5 & 104.3 \\
\hline 6471.668 & 20.0 & 2.520 & -0.690 & 102.3 & 110.0 & 108.8 & 131.6 & 111.1 \\
\hline 6481.878 & 26.0 & 2.280 & -2.940 & 113.4 & 95.1 & 96.5 & 108.5 & 101.3 \\
\hline 6482.809 & 28.0 & 1.930 & -2.780 & 79.4 & 78.7 & - & 79.7 & 68.9 \\
\hline 6493.788 & 20.0 & 2.520 & -0.110 & 143.4 & 134.0 & 133.3 & 146.2 & 132.7 \\
\hline 6496.908 & 56.1 & 0.600 & -0.380 & 153.5 & 141.7 & 142.2 & 200.6 & - \\
\hline 6498.945 & 26.0 & 0.960 & -4.660 & 112.4 & 87.1 & 97.1 & 101.3 & 84.5 \\
\hline 6499.654 & 20.0 & 2.520 & -0.820 & 103.6 & 114.1 & 105.0 & 121.0 & 96.9 \\
\hline 6516.083 & 26.1 & 2.890 & -3.380 & 68.0 & 71.6 & - & 104.8 & 95.2 \\
\hline 6518.373 & 26.0 & 2.830 & -2.560 & 128.8 & 77.7 & 81.6 & 104.2 & - \\
\hline 6533.940 & 26.0 & 4.560 & -1.200 & - & 57.0 & - & 62.8 & - \\
\hline 6554.238 & 22.0 & 1.440 & -1.220 & 88.5 & 51.7 & - & 41.8 & - \\
\hline 6572.795 & 20.0 & 0.000 & -4.320 & 135.9 & 94.1 & 89.8 & 79.1 & 57.0 \\
\hline 6574.254 & 26.0 & 0.990 & -4.960 & 123.9 & 83.4 & 91.6 & 85.3 & 60.0 \\
\hline 6581.218 & 26.0 & 1.480 & -4.680 & - & 65.3 & 51.2 & 60.4 & 52.9 \\
\hline 6586.319 & 28.0 & 1.950 & -2.780 & 80.6 & 64.7 & 66.8 & 76.6 & 59.2 \\
\hline 6593.884 & 26.0 & 2.430 & -2.300 & 118.0 & 136.0 & - & 128.1 & 113.7 \\
\hline 6598.611 & 28.0 & 4.230 & -0.930 & - & - & - & 38.5 & - \\
\hline 6604.600 & 21.1 & 1.360 & -1.140 & - & 73.6 & - & 85.1 & 86.3 \\
\hline 6606.979 & 22.1 & 2.060 & -2.900 & - & - & - & 35.5 & - \\
\hline 6608.044 & 26.0 & 2.280 & -3.960 & - & 52.4 & - & 47.0 & 30.6 \\
\hline 6609.118 & 26.0 & 2.560 & -2.650 & - & 98.7 & 105.1 & 105.8 & 92.0 \\
\hline 6625.039 & 26.0 & 1.010 & -5.320 & 90.6 & 55.3 & 46.8 & 57.9 & 39.7 \\
\hline 6627.560 & 26.0 & 4.550 & -1.500 & - & 36.0 & 44.0 & 50.0 & 33.9 \\
\hline 6633.758 & 26.0 & 4.560 & -0.810 & 63.7 & 82.4 & 65.4 & - & - \\
\hline 6635.137 & 28.0 & 4.420 & -0.750 & - & 39.7 & - & 41.2 & 21.1 \\
\hline 6696.032 & 13.0 & 3.140 & -1.320 & - & 58.0 & - & 57.6 & 50.8 \\
\hline 6698.669 & 13.0 & 3.140 & -1.620 & - & 28.6 & - & - & - \\
\hline 6703.576 & 26.0 & 2.760 & -3.000 & 87.9 & 69.9 & 47.8 & 77.8 & 55.2 \\
\hline 6713.745 & 26.0 & 4.790 & -1.410 & - & 34.7 & - & 36.1 & 22.9 \\
\hline 6725.364 & 26.0 & 4.100 & -2.210 & 48.5 & 27.1 & - & 33.0 & 23.5 \\
\hline 6726.673 & 26.0 & 4.610 & -1.050 & 41.8 & 67.5 & 45.6 & 59.8 & 53.1 \\
\hline 6733.153 & 26.0 & 4.640 & -1.440 & - & - & - & 44.0 & 31.0 \\
\hline 6739.524 & 26.0 & 1.560 & -4.850 & 72.8 & 48.5 & - & 37.2 & 25.4 \\
\hline 6750.164 & 26.0 & 2.420 & -2.580 & - & 112.2 & 93.9 & 126.3 & 104.7 \\
\hline 6767.784 & 28.0 & 1.830 & -2.060 & 124.8 & 119.0 & 83.7 & 119.0 & 105.8 \\
\hline 6772.321 & 28.0 & 3.660 & -0.960 & 61.1 & 68.4 & 55.5 & 74.9 & 54.7 \\
\hline 6786.860 & 26.0 & 4.190 & -1.900 & 62.2 & 39.0 & - & 40.7 & 29.5 \\
\hline
\end{tabular}

